VOLUME 36, ARTICLE 54, PAGES 1667-1702 PUBLISHED 24 MAY 2017

http://www.demographic-research.org/Volumes/Vol36/54/

DOI: $\quad$ 10.4054/DemRes.2017.36.54

Research Article

\title{
How many old people have ever lived?
}

\section{Miguel Sánchez-Romero}

\section{Dalkhat Ediev}

Gustav Feichtinger

Alexia Prskawetz

(C) 2017 Sánchez-Romero, Ediev, Feichtinger \& Prskawetz.

This open-access work is published under the terms of the Creative Commons Attribution NonCommercial License 2.0 Germany, which permits use, reproduction \& distribution in any medium for non-commercial purposes, provided the original author(s) and source are given credit. See http://creativecommons.org/licenses/by-nc/2.0/de/ 


\section{Contents}

$1 \quad$ Introduction $\quad 1668$

$2 \quad$ Analytical framework and empirical assessments $\quad 1669$

$\begin{array}{lll}2.1 & \text { Analytical framework } & 1669\end{array}$

$\begin{array}{lll}2.2 & \text { Empirical assessments } & 1671\end{array}$

$3 \quad$ Formal population models 1674

$3.1 \quad$ Exponential population growth 1675

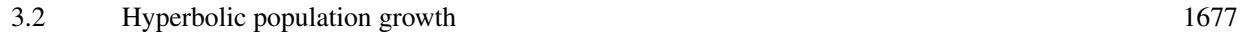

$4 \quad$ Dynamic features of $\pi(y, T) \quad 1678$

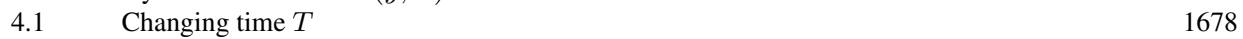

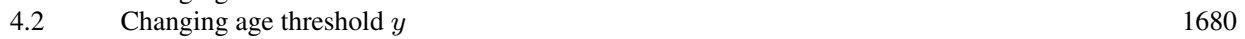

$\begin{array}{lll}5 & \text { Conclusion and discussion } & 1684\end{array}$

6 Acknowledgements 1686

$\begin{array}{ll}\text { References } & 1687\end{array}$

$\begin{array}{ll}\text { Appendix A } & 1689\end{array}$

$\begin{array}{lr}\text { Appendix B } & 1690\end{array}$

$\begin{array}{ll}\text { Appendix C } & 1693\end{array}$

$\begin{array}{ll}\text { Appendix D } & 1699\end{array}$ 


\title{
How many old people have ever lived?
}

\author{
Miguel Sánchez-Romero ${ }^{1}$ \\ Dalkhat Ediev ${ }^{2}$ \\ Gustav Feichtinger ${ }^{3}$ \\ Alexia Prskawetz ${ }^{4}$
}

\begin{abstract}
BACKGROUND

Uninformed generalizations about how many elderly people have ever lived, based on a poor understanding of demography, are found in a surprising number of important publications.
\end{abstract}

\section{OBJECTIVE}

We extend the methodology applied to the controversial question "how many people have ever been born?" initiated by Fucks, Winkler, and Keyfitz, to the proportion of people who have ever reached a certain age $y$ and are alive today (denoted as $\pi(y, T)$ ).

\section{METHODS}

We first analyze the fraction $\pi(y, T)$ by using demographic data based on UN estimates. Second, we show the main mathematical properties of $\pi(y, T)$ by age and over time. Third, we complete our analysis by using alternative population models.

\section{RESULTS}

We estimate that the proportion who have ever been over 65 that are alive today (as of 2010) ranges between 5.5 and $9.5 \%$. We extend the formal demographic literature by considering the fraction of interest in two frequently referred models: the stable and hyperbolic growth populations.

\footnotetext{
${ }^{1}$ Wittgenstein Centre for Demography and Global Human Capital (IIASA,VID/ÖAW, WU). E-Mail: miguel.sanchez@oeaw.ac.at.

${ }^{2}$ North Caucasian State Humanitarian Technological Academy (IAMIT), Lomonosov Moscow State University (HSMSS/Demography Department), Wittgenstein Centre for Demography and Global Human Capital (IIASA,VID/ÖAW, WU). E-Mail: ediev@iiasa.ac.at.

${ }^{3}$ Vienna University of Technology (TU Wien), Wittgenstein Centre for Demography and Global Human Capital (IIASA,VID/ÖAW, WU). E-Mail: gustav.feichtinger@tuwien.ac.at.

${ }^{4}$ Vienna University of Technology (TU Wien), Wittgenstein Centre for Demography and Global Human Capital (IIASA,VID/ÖAW, WU). E-Mail: afp@econ.tuwien.ac.at.
} 
Sánchez-Romero et al.: How many old people have ever lived?

\section{CONCLUSIONS}

We show that statements claiming half of all people who have ever reached the age of 65 are alive today ranges would never be attainable, neither theoretically nor empirically, according to existing data.

\section{CONTRIBUTION}

We have produced for the first time a harmonized reconstruction of the human population by age throughout history. For a given contemporaneous time $T$, we demonstrate analytically and numerically that $\pi(y, T)$ is nonmonotonic in age $y$. For a given age $y$, we show that $\pi(y, T)$ may also be nonmonotonic with respect to $T$.

\section{Introduction}

Global population ageing, caused by fertility decline and increasing survival at older ages, has become a challenging issue of our times. The shift in the age structure of the population will profoundly reshape the social structure of our world as well as its economy.

There are around 600 million people aged 65 or older alive today. While their share is now about $8 \%$ of the total population, it will increase to some $13 \%$ in the next 20 years. According to the UN's population projections the world had 16 people aged 65 and over for every 100 adults between the ages of 25 and 64, but this dependency ratio will rise to 26 by 2035 .

A recent article in the Economist describes how those "age invaders" are about to change the global economy. Besides the old age dependency ratio, in this publication another indicator of aging is mentioned: The ratio 65 or older alive today relative to all humans who have ever reached the age of 65. According to the Economist, Fred Pearce presumed that it is possible that half of all people who have ever been over 65 are alive today. Motivated by these discussions, in our paper we reconsider indicators that estimate the share of people above a specific age alive today in relation to all the humans who have ever reached this specific age. By using formal demography together with historical data on population processes, we show how such indicators can be estimated. Our results indicate that far fewer than half of all people who have ever been over 65 are alive in 2010.

Clearly, this paper is closely related to a question that has been posed by several prominent demographers, namely "How many people have ever lived on earth?" In his seminal book Applied Mathematical Demography, Keyfitz (1977) gives a brief introduction to the problem. Among the demographers who have dealt with this problem are Petty 
(1682), Winkler (1959), Deevey (1960), Desmond (1962), and Keyfitz (1966). More recent references are Tattersall (1996), Johnson (1999), Haub (2011), and Cohen (2014).

Cohen (2014) shows a table with various estimates of the number of people ever born by year $t$ starting with Petty (1682) until Haub (2011). It illustrates the wide range of the various estimates. For instance Haub's (2011) semi-scientific approach yields an estimate of 108 billion births since the dawn of the human race, assumed as 50,000 B.C. Thus $6.5 \%$ of those ever born were living in mid-2011.

Asking the question whether this fraction rises or falls, Cohen (2014) comes to the robust conclusion that at present it is increasing. On the other hand, if world population were to reach stationarity or decline, the fraction would fall. The significance of Cohen's analysis lies in the fact that he uses mathematical demography to obtain his results. The present paper follows his reasoning. By extending his approach we study the fraction of people ever surpassing a certain age limit $y$, say 65 years, who are now alive.

The paper is organized as follows. In Section 2 we introduce an analytic expression of the ratio of the number of people at ages above $y$ in year $T$ to the number of those that ever reached the age $y$ and present a first rough and a more refined estimate of this number based on given historical population estimates. In Section 3 we analyze the behavior of $\pi(y, T)$ under different formal population models. In particular, we apply an exponential growth model (i.e., stable population) and alternatively a hyperbolic population model. Section 4 is devoted to an analytic and numerical investigation of the dynamic change in this expression with respect to the age threshold $y$ and the time $T$. The final section concludes and highlights how far off estimations of our expression could be by using wrong models of historical populations.

\section{Analytical framework and empirical assessments}

In this section we first present the general formula to calculate the fraction of people over age $y$ who ever lived who are currently alive in year $T$, which we denote by $\pi(y, T)$. Second, we calculate using data from several authors the ratio of people at age 65 alive in 2010 to the number of those who ever reached age 65 .

\subsection{Analytical framework}

Let $N(a, t)$ be the population size at age $a$ in year $t ; B(c)$ be the number of births in year $c$; and $\ell(a, c)$ be the survival probability to age $a$ for the birth cohort $c$. The number of people that ever reached old age $y$ since the original cohort $c=0$ is:

$$
\int_{0}^{T-y} N(y, c+y) d c=\int_{0}^{T-y} B(c) \ell(y, c) d c
$$


Sánchez-Romero et al.: How many old people have ever lived?

while the number of people currently alive at ages $y$ and older is (assuming $T>\omega$, where $\omega$ is the maximum age):

$$
\int_{y}^{\omega} N(a, T) d a=\int_{T-\omega}^{T-y} B(c) \ell(T-c, c) d c .
$$

The proportion of interest is the ratio of the number of people currently at ages $y+$ to the number of those ever reached the age $y$ :

$$
\pi(y, T)=\frac{\int_{y}^{\omega} N(a, T) d a}{\int_{0}^{T-y} N(y, c+y) d c}=\frac{\int_{T-\omega}^{T-y} B(c) \ell(T-c, c) d c}{\int_{0}^{T-y} B(c) \ell(y, c) d c} .
$$

The numerator of Equation (3) accounts for the living population older than age $y$ in year $T$, which is represented by the vertical solid line in Figure 1, while the denominator of Equation (3) is the population that ever lived to age $y$ until year $T$, or the solid horizontal line in Figure 1.

Figure 1: $\quad$ Lexis diagram illustrating the calculations of $\pi(y, T)$

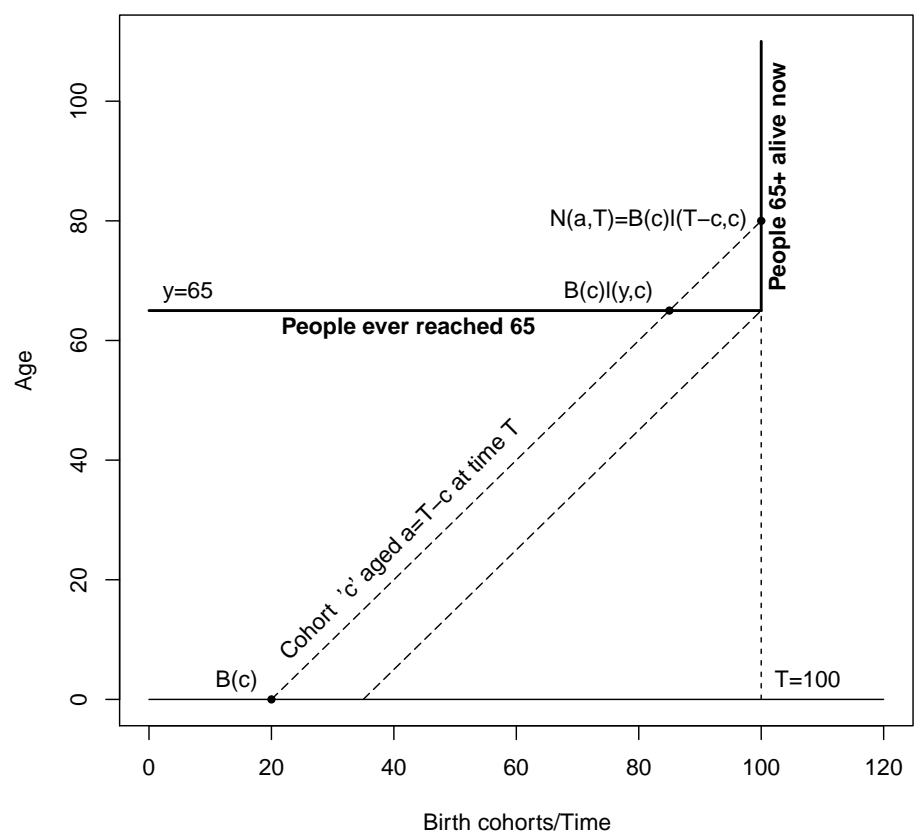




\subsection{Empirical assessments}

Up to now Equation (3) has been empirically estimated several times since the pioneering article by Fucks (1951) for age $y$ equal to zero. However, to our knowledge, no one has ever rigorously estimated the value of $\pi(y, T)$ for an age $y$ greater than zero. In this section we present the first estimations of $\pi(y, 2010)$ for an age $y$ equal to 65 using two different approaches. One approach is based on breaking human history into several time intervals and assuming that the population grew at a constant rate within each interval. In our second approach we relax the assumption of a constant population growth within each time interval.

For a first estimate of $\pi(65,2010)$, we took data on total population and births before 1945 from Deevey (1960), Keyfitz (1966), Westing (1981), and Haub (2011). These four authors cover plausible minimum (5.5\%) and maximum (13.9\%) values of the people who ever lived to age 65 who are alive in 2010. In all papers, the births born are calculated by dividing human history into several time intervals, within which the population is assumed to grow at a constant rate. Differences in the number of people who ever lived among all authors stem mainly from the number of intervals used, the assumed life expectancy at birth, and the crude birth rate in the first periods. ${ }^{5}$

For instance, the number of time intervals up to 1945 used by Deevey (1960) is 11, 8 intervals are applied by Haub (2011), 6 intervals by Westing (1981), and 4 intervals by Keyfitz (1966). In the first time intervals, the life expectancy at birth ranges between age 13 (Haub 2011) and 25 (Deevey 1960; Keyfitz 1966), with a middle value of 20 assumed by Westing (1981). To compute the number of people that ever lived to age 65 , shown in Table 1, we multiply the total population born by the corresponding survival probability to age 65 in each period. The values of the survival probability to age 65 by different life expectancy are drawn from the UN General Model Life Table. See Table A-1 in the Appendix D for the calculations performed for each author.

\footnotetext{
${ }^{5}$ Recall that in a stable population, for a given population growth rate there exists in a single parametric family of life tables a one-to-one relationship between life expectancy at birth and crude birth rate.
} 
Sánchez-Romero et al.: How many old people have ever lived?

Table 1: $\quad$ Fraction of people who ever lived to age 65 and were alive in 2010

\begin{tabular}{lrrrr}
\hline & Deevey (1960) & Westing (1981) & Keyfitz (1966) & Haub (2011) \\
\hline Persons ever born until 1945 (millions) $^{\dagger}$ & 83,719 & 45,951 & 67,138 & 99,803 \\
Persons age 65 ever lived (millions) $^{\ddagger}$ & 9,575 & 7,991 & 6,640 & 3,762 \\
Persons age 65+ in 2010 (millions) & 524 & 524 & 524 & 524 \\
$\pi(65,2010)$ & 0.055 & 0.066 & 0.079 & 0.139 \\
\hline
\end{tabular}

Source: † Data collected from Johnson (1999). $\ddagger$ Author’s calculations based on UN Model Life Tables by life expectancy and people who ever lived collected by Johnson (1999). ' Data taken from the United Nations Department of Economic and Social Affairs, Population Division (UN 2013).

These assessments led to the estimate that the number of people who have survived to age 65 until 2010 ranges between 3,762 and 9,575 million people. The lowest value, obtained by Haub (2011), crucially depends on a low life expectancy even for the most recent decades, while the highest value obtained by Deevey (1960) is due to the combination of a long time span (i.e., more than one million years) together with a high initial population size (i.e., 125,000 people). Given that the UN estimates a total number of people age $65+$ in 2010 at close to 524 million, we obtain that between $5.5 \%$ and $13.9 \%$ of the total population who ever reached age 65 were alive in 2010. It is clear that these values fall below the presumption that half of people who have ever been over age 65 are alive today.

Unlike the previous estimate, in our second approach we more realistically assume that fertility and mortality vary within each time interval. As a consequence, this assessment better accounts for the rapid change in the vital rates during the last century. We do so by using a Generalized Inverse-Projection (GIP) model, which allows us to reconstruct the historical population by taking as a priori information the population numbers used in Table 1 (Lee 1966; Oeppen 1993). More importantly, the GIP model allows us to match the reconstructed populations until 1950 with population data from 1950 to 2100 estimated by the UN (2013). ${ }^{6}$ The match of the historical population to this UN data from 1950 to 2010 can be seen in Figure A-4 in Appendix C. The population numbers will be used in Section 4 to illustrate the dynamic features of $\pi(y, T)$. See Appendix $\mathrm{C}$ for the model details.

Table 2 shows the total number of people age 65 who ever lived from 50,000 B.C until 2010 A.C that results from using in the GIP model the population data of Haub (2011) - column 3 - and Deevey (1960) - column 5.

\footnotetext{
${ }^{6}$ Note that combining population numbers that result from a stable population model with UN (2013) data, which is clearly unstable, would have caused misleading results because of artificial jumps in $\pi(y, T)$.
} 
Table 2: $\quad$ Number of people age 65 who ever lived (in millions)

\begin{tabular}{rrrrr}
\hline Year t & Population & $\begin{array}{c}\text { Haub (2011) } \\
\text { Pop. age 65 } \\
\text { who ever lived }\end{array}$ & Population & $\begin{array}{c}\text { Deevey (1960) } \\
\text { Pop. age 65 } \\
\text { who ever lived }\end{array}$ \\
\hline-50000 & 0 & 0 & 3 & 1,921 \\
-8000 & 5 & 36 & 6 & 2,404 \\
1 & 309 & 1,547 & 139 & 4,699 \\
1200 & 432 & 2,350 & 369 & 5,882 \\
1650 & 516 & 2,823 & 544 & 6,743 \\
1750 & 800 & 3,003 & 732 & 7,006 \\
1850 & 1,277 & 3,342 & 1,199 & 7,389 \\
1900 & 1,681 & 3,620 & 1,637 & 7,678 \\
1950 & 2,587 & 4,118 & 2,577 & 8,126 \\
1970 & 3,758 & 4,422 & 3,760 & 8,427 \\
1990 & 5,354 & 4,861 & 5,361 & 8,869 \\
2000 & 6,177 & 5,159 & 6,184 & 9,168 \\
2005 & 6,573 & 5,330 & 6,579 & 9,340 \\
2010 & 6,896 & 5,514 & 6,896 & 9,524 \\
\hline
\end{tabular}

Source: Haub (2011), Deevey (1960), are used until 1900 and UN (2013) is used from 1950 to 2010.

These assessments give a total number of people who have survived to age 65 through 2010 as ranging from 5,514 to 9,524 million people (see the last row in Table 2). Therefore, if the population older than 65 in 2010 was 524 million people, $\pi(65,2010)$ ranges between $5.5 \%$ and $9.5 \%$. The difference between the first empirical assessment and the refined assessment for Haub (2011) stems from the fact that in the latter, more people survived to age 65 because the rapid mortality improvements during the last half of the $20^{\text {th }}$ century are taken into account. Similarly, we do not observe a large difference between the assessments made for Deevey's (1960) population data because the life expectancies assumed in the last intervals are closer to the actual values.

The GIP model also provides interesting additional insights. For instance, Figure 2 shows the persons-years ever lived up to each age until 2010 based on different historical population data. In panel 2 the absolute numbers are provided and we can see the comparison between the relative shares of the persons-years ever lived across age to the population distribution in $2010 .^{7}$. Given that under a stable population the current population distribution should coincide with the relative size of the persons-years ever lived, Figure 2 gives us information about the pace of aging of the population.

In particular, based on Figure 2 the average age of the total population in 2010 was 30.9 years, while the average age of the people who ever lived up to 2010 is either 22.3 or 25.9, assuming Haub's or Deevey's population data, respectively. Thus, this result provides us information about the unusual stage that the population is facing and how the pace of ageing is increasing.

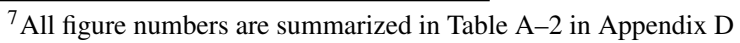


Sánchez-Romero et al.: How many old people have ever lived?

\section{Figure 2: $\quad$ Persons-years ever lived up to 2010}
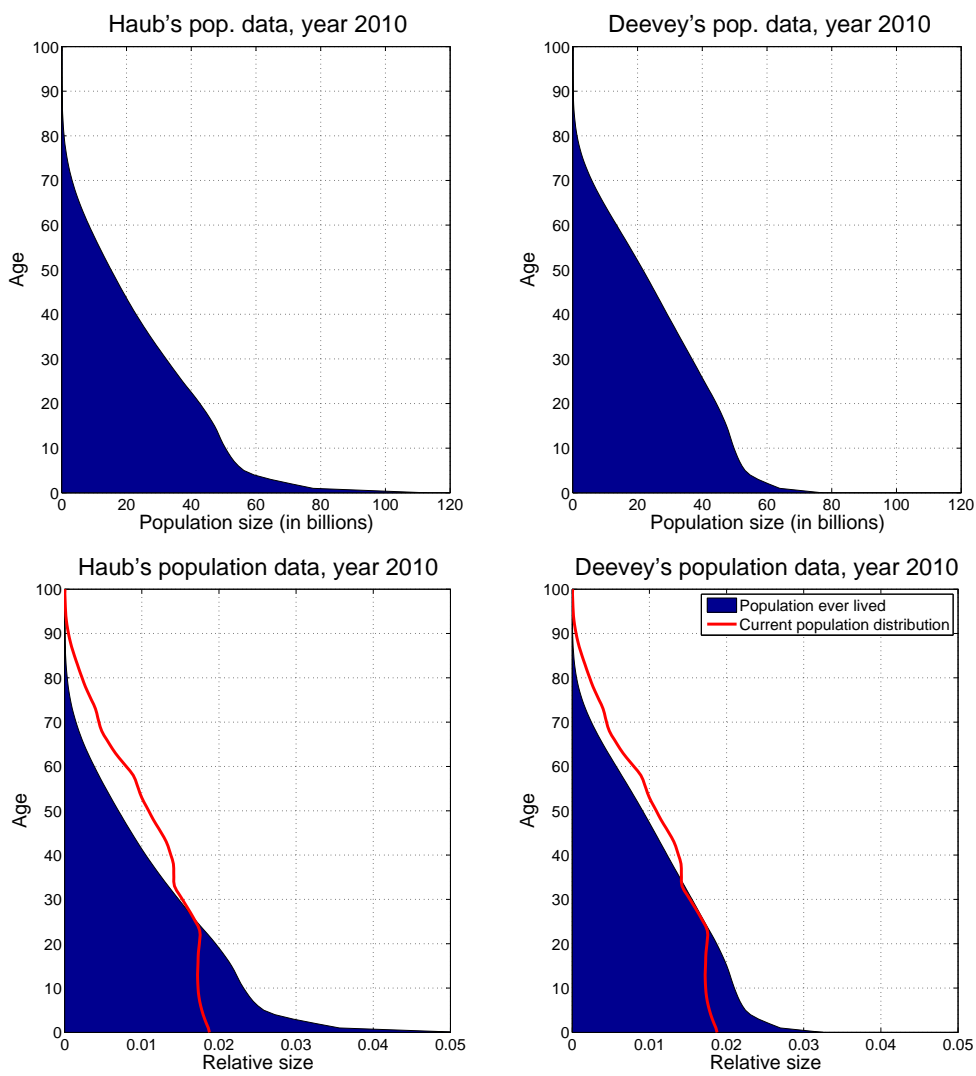

\section{Formal population models}

In this section, we study the concept of population ever lived to a given age under two common population models. Such a formal demographic approach allows us to derive analytical expressions of our indicator of interest. First, we consider the classical model where numbers of births and all population numbers grow in exponential fashion consistent with time-invariant fertility and mortality rates, the model referred to as the stable population (Keyfitz and Caswell 2005; Preston, Heuveline, and Guillot 2001). Because the population growth rate historically increased over time, the exponential mode overestimates person-years in the past and therefore produces a lower estimate for the pro- 
portion of people who ever lived who are alive now. The other model considered here, the hyperbolic growth model, assumes that the population growth rate is proportional to population size. As a consequence, the hyperbolic model leads to a higher estimate of the proportion of people who ever lived who are alive now. These two models provide useful formal demographic boundaries to the proportion of interest. Our results also contribute to a better understanding of the two important formal demographic models of population growth, i.e., which of these two models might better approximate demographic numbers such as the population who ever lived to a given age.

\subsection{Exponential population growth}

In the simplest case of a stable population, where life tables are assumed to be constant across cohorts (i.e., $\ell(a, c)=\ell(a))$ and births are assumed to grow exponentially at a constant rate $r$ (i.e., $\left.B(c)=B(0) e^{r c}\right), \pi(y, T)$ becomes

$$
\begin{cases}\frac{r}{1-e^{-r(T-y)}} \int_{y}^{\omega} e^{-r(a-y) \frac{\ell(a)}{\ell(y)} d a} & \text { if } r \neq 0 \\ \frac{1}{T-y} \int_{y}^{\omega} \frac{\ell(a)}{\ell(y)} d a & \text { if } r=0 .\end{cases}
$$

The integral in Equation (4) is the stable population at ages $y+$ divided by the stable population of exact age $y$, while the fraction in front of the integral is the ratio between the total births born in year $T-y$ and the person-years lived between 0 and $T-y$.

In a stable population the fraction $\pi(y, T)$ converges to zero for $r \leq 0$. Assuming positive population growth and $T \gg y$, the ratio converges to the limit value:

$$
\pi(y, T)=r \int_{y}^{\omega} e^{-r(a-y)} \frac{\ell(a)}{\ell(y)} d a .
$$

Hence, under a stable population, the value of the integral is given by the inverse of the proportion of people aged 65 divided by those age $65+$, which according to the UN estimates is about $7.50 \%$ at age $y=65$ in 2010. On the other hand, the geometric mean of the long-run population growth rate from the origin of our race $(50,000 \mathrm{BC})$ is approximately $0.035 \%$. Consequently, if we use the existing data and assume a stable population model, the value of $\pi(65,2010)$ will be $\frac{0.00035}{0.075} \simeq 0.47 \%$, which according to Figure 3 is above the range $0.20-0.35 \%$ that is obtained if a stable population with a life expectancy at birth between age 20 and 40 , respectively, is assumed. 
Sánchez-Romero et al.: How many old people have ever lived?

\section{Figure 3: $\quad$ Ratio of people age 65+ who are alive in 2010 to people who ever lived to age 65, by life expectancy at birth and growth rate of births}

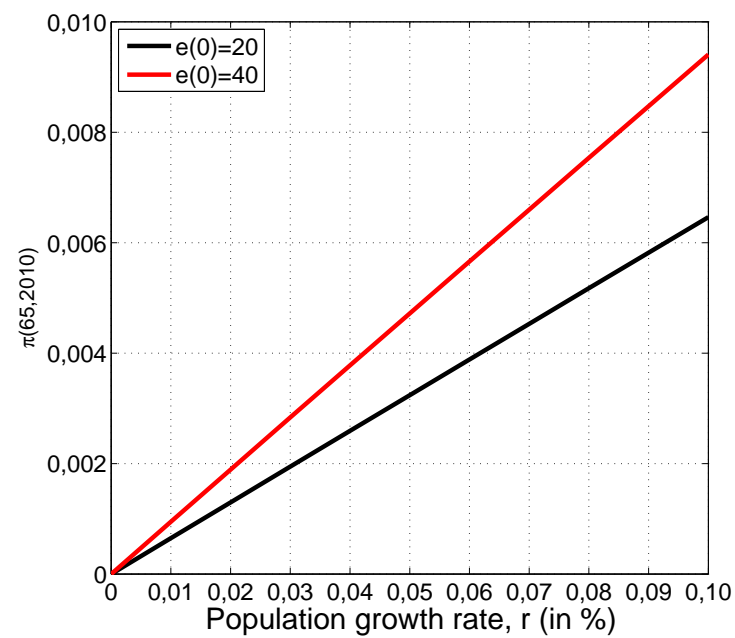

Note: Survival probabilities by life expectancy taken from the UN General Model Life Tables.

This result shows that the stable population model is not capable of reproducing well the empirically assessed values of $\pi(65,2010)$ shown in Section 2 . Partly this is because the exponential growth model does not account for recent improvements in survival to old age. However, even if we use an expression that takes into account actual data on current population size and age composition, this model yields a low value of $\pi(65,2010){ }^{8}$ Partly this is because the low historical growth rate produces a high number of people ever born relative to those who are currently alive. Thus, if we fit the exponential growth model to more recent data with faster population growth, the number of people ever born would be too low and the proportion $\pi(65,2010)$ too high. Fitting the model to the growth of population aged 65 from 1950 until 2010 (UN estimates) yields the estimate of about 1,953 million people who ever lived to age 65 . Combining this estimate with the current number of people aged $65+$ gives a $\pi(65,2010)$ value equal to $26.8 \%$, which is above the more accurate empirical assessments of the previous section (but still well

${ }^{8}$ Assuming a stable population, we can calculate $\pi(y, T)$ using only current population data as follows

$$
\pi(y, T)=\frac{\int_{y}^{\omega} N(a, T) d a}{\int_{0}^{T-y} N(y, c+y) d c}=\frac{\int_{y}^{\omega} N(a, T) d a}{N(y, T) \int_{0}^{T-y} e^{-r(T-y-c)} d c} .
$$

Note that this expression reflects the recent mortality decline. 
below the $50 \%$ level). In sum, the estimates based on the exponential growth model are too sensitive to the growth rate assumed in the model, and a single growth rate may not fit well to the actual population history with many periods of accelerated population growth (Keyfitz 1966).

\subsection{Hyperbolic population growth}

The inability of the exponential growth model to fit the historically varying growth rate led researchers to super-exponential models (von Foerster, Mora, and Amiot 1960; von Hoerner 1975; Kapitza 1992; Kremer 1993), where the growth rate increases in relation to a stock population variable. ${ }^{9}$ Here we consider one particular type of such a model, the hyperbolic growth where (to better account for the varying vital rates affecting a specific age, we write the model for the population size at age $y$ and not the total population size)

$$
\frac{1}{N(y, t)} \frac{\partial N(y, t)}{\partial t}=\alpha N(y, t) \text {. }
$$

Solving this equation leads to

$$
N(y, t)=N(y, 0) \frac{\tau}{\tau-t} \text { for any } t<\tau,
$$

where $N(y, 0)$ is the population size of age $y$ at the onset of the hyperbolic growth and $\tau=\frac{1}{\alpha N(y, 0)}$ is the time when the model produces a vertical asymptote. Integrating the number of people at age $y$ until year $T$ gives

$$
\int_{0}^{T} N(y, t) d t=N(y, T)(\tau-T) \log \frac{\tau}{\tau-T} .
$$

Therefore, the ratio of the number of people currently at ages $y+$ to the number of those ever reached age $y$ is:

$$
\pi(y, T)=\frac{1}{(\tau-T) \log \frac{\tau}{\tau-T}} \int_{y}^{\omega} \frac{N(a, T)}{N(y, T)} d a .
$$

Applying the hyperbolic growth model to the population at age 65 , and fitting the model to the empirical numbers $N(65,2010)=39.1$ million and $N(65,1950)=12.8$ million yields $\tau=2039$ AC. Realize that the value $\tau$ would get closer to $T$ when longer time intervals are used. Then, given that the UN estimates a total number of people age 65+ of 524.4 million people, Table 3 shows the following estimates for the people who ever reached age 65 and $\pi(65,2010)$ :

\footnotetext{
${ }^{9}$ To account for the acceleration of the population growth rate, Cohen (2014) also uses a super-exponential model.
} 
Sánchez-Romero et al.: How many old people have ever lived?

Table 3: $\quad$ Number of people age 65 ever lived: Hyperbolic model

\begin{tabular}{lrrrrrr}
\hline$\tau$ & 2015 & 2030 & 2039 & 2045 & 2060 & 2075 \\
\hline Persons age 65 ever lived (millions) & 1,807 & 6,145 & 8,489 & 9,988 & 13,573 & 16,979 \\
Persons age 65+ in 2010 (millions) & 524 & 524 & 524 & 524 & 524 & 524 \\
$\pi(65,2010)$ & 0.290 & 0.085 & 0.062 & 0.052 & 0.039 & 0.031 \\
\hline
\end{tabular}

Note: Numbers are calculated assuming 50000 B.C as our initial year.

Note in Table 3 that as $\tau$ gets closer to $T=2010$, the model produces very rapid population growth rates and hence smaller values for the number of people who ever reached age 65 . Nevertheless, even when $\tau=2015, \pi(65,2010)$ is notably well below the $50 \%$ level. Fitting the model to the recent past, i.e., by setting $\tau=2030$, the model yields $\pi(65,2010)=6.2 \%$. This rate is between the range of plausible values obtained in the refined assessment $(5.5 \%-9.5 \%)$ and higher than the exponential population growth model.

\section{Dynamic features of $\pi(y, T)$}

An analytical study of the dynamics of the ratio $\pi(y, T)$ is key to understanding its plausible boundaries. It also provides the necessary tools for analyzing in a systematic way past, present, and future values of $\pi(y, T)$. Recently, Cohen (2014) has shown that $\pi(0, T)$ (i.e., the fraction of people ever born up to time $T$ who are alive at time $T$ ) decreases over time for a stable population model, but it can increase or decrease with a superexponential or with a doomsday model. In this section we extend the analysis of Cohen (2014) by studying the dynamic features of the new indicator $\pi(y, T)$. Moreover, we provide values for $\pi(y, T)$ across different ages and over time using actual world population projections. Since $\pi(y, T)$ is a two dimensional function, we explore the change of $\pi(y, T)$ over time and over the threshold age $y$. Thus, we first differentiate $\log \pi(y, T)$ with respect to time and, second, with respect to the threshold age $y$.

\subsection{Changing time $T$}

To analyze whether $\pi(y, T)$ might reach values close to $50 \%$ in the near future, we differentiate $\log \pi(y, T)$ with respect to time $T$. After rearranging terms, we obtain ${ }^{10}$

$$
\frac{\pi_{T}(y, T)}{\pi(y, T)}=\frac{N(y, T)-\int_{y}^{\omega} N(a, T) \mu(a, T) d a}{\int_{y}^{\omega} N(a, T) d a}-\frac{N(y, T)}{\int_{0}^{T-y} N(y, c+y) d c} .
$$

\footnotetext{
${ }^{10}$ For an illustration of the derivative of $\pi(y, T)$ with respect to $T$, see Figure A-1 in Appendix A.
} 
Equation (11) is the difference between the fractional change over time in the number of people alive above age $y$ and the fractional change over time in the number who have ever reached age $y$. Equation (11) coincides with Equation (2) in Cohen (2014), page 1562 , when $y=0$. The fractional change over time in the number of people alive above age $y$ in year $T$ can be either positive or negative. Indeed, the first term is the crude growth rate in year $T$ of the population older than age $y$. In contrast, the second term in Equation (11) is always negative. As a result, $\pi(y, T)$ can either increase or decrease over time. Another important difference is that the first term in Equation (11) depends only on current information, whereas the second term depends on the historical population.

Assuming a stable population, we know from Proposition 1 that $\pi(y, T)$ is a decreasing function with respect to time $T$ (see proof in Appendix A), which converges in the limit to Equation (5).

Proposition 1. In a stable population, for all $r, \pi(y, T)$ is monotonically decreasing with respect to time $T$.

Proposition 1 implies that for any stable population growth rate $r$, our fraction of interest $\pi(y, T)$ decreases over time at any age threshold $y$. This proposition extends to any arbitrary age $y$ the result of Cohen (2014) for a stable population. The fact that $\pi(y, T)$ monotonically decreases over time is explained by two properties. First, in a stable population, the number of people currently alive at age $y+$, or numerator, increases at the same rate as the population. Second, in a stable population, the growth rate of the number of people who ever reached the age $y$, or denominator, is also positive but, when the time horizon is finite $(0, T)$, it increases at a decreasing rate over time. Thus, when $T$ tends to infinity, the growth rate of the number of people who ever reached age $y$ asymptotically converges to the population growth rate. This explains why $\pi(y, T)$ starts at $T=\omega$ at a high value and monotonically decreases towards Equation (5).

Figure 4 illustrates Proposition 1 by plotting all possible values of $\pi(y=65, T)$ between year $T=\omega$ and $T \uparrow \infty$ by different life expectancies at birth and growth rates of births. All feasible values are contained in the blue area. Since $\pi(y, T)$ decreases over time, the highest value of $\pi(65, T)$ for a given population growth rate occurs when $T=\omega$, while the lowest value occurs when $T \uparrow \infty$. Figure 4 also provides two interesting results. First, higher population growth rates lead to higher values of $\pi(y, T)$. Second, a higher life expectancy also increases the value of $\pi(y, T)$. Therefore, given that during the demographic transition both fertility and mortality changes, the sign of Equation (11) is a priori ambiguous and we need to perform an empirical analysis. Nevertheless, the first term in Equation (11) will typically be higher than the second one when the growth rate of births increases, because the population reaching age $y$ increases faster than the deaths above that age (Cohen 2014). For this reason, as shown in Figure 5, $\pi(y, T)$ has continuously increased during the $20^{\text {th }}$ century at all ages analyzed. In the $21^{s t}$ century, however, according to the medium variant UN (2013) estimates, the proportion $\pi(y, T)$ 
Sánchez-Romero et al.: How many old people have ever lived?

may eventually decline at different ages after reaching a maximum due to the expected slowdown in the growth rate of births.

Figure 4: $\quad$ Ratio of people age 65+ who are alive in year $T$ to people who ever lived to age 65 until year $T$, by life expectancy at birth and growth rate of births

(a) Life expectancy (at birth) $=20$

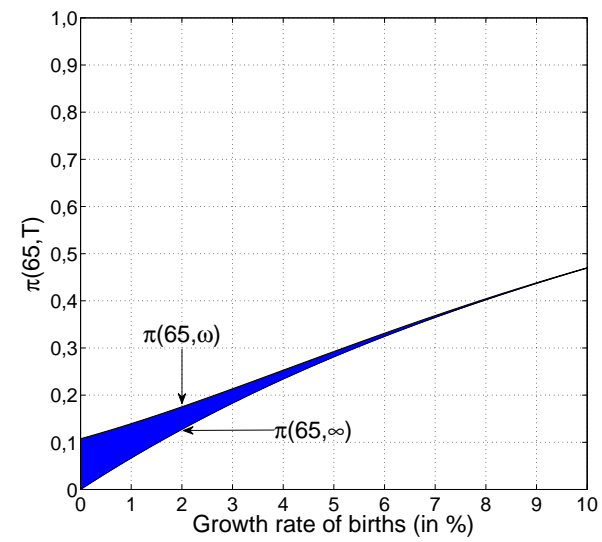

(b) Life expectancy (at birth) $=80$

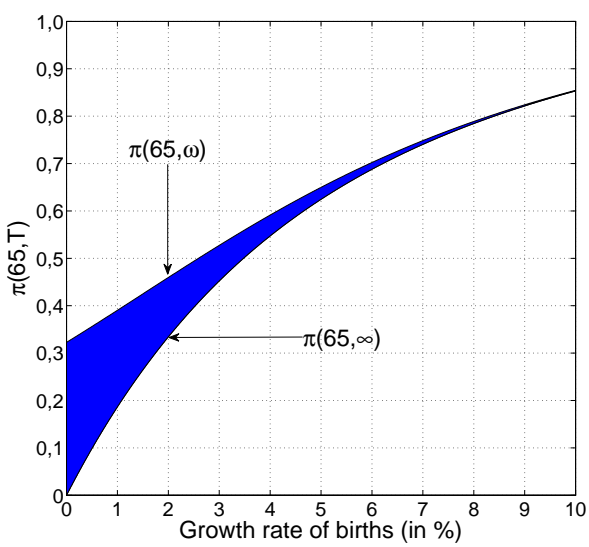

Note: Survival probabilities by life expectancy taken from the UN General Model Life Tables.

For instance, $\pi(0, T)$ is expected to reach a maximum value between $8-12 \%$ during the second half of the $21^{\text {st }}$ century, $\pi(65, T)$ will peak between $13-19 \%$ in the $2060 \mathrm{~s}$.

\subsection{Changing age threshold $y$}

In the first case, taking logarithms of both sides of Equation (3) and differentiating with respect to $y$ gives

$$
\frac{\pi_{y}(y, T)}{\pi(y, T)}=\frac{N(y, T)+\int_{0}^{T-y} N(y, c+y) \mu(y, c+y) d c}{\int_{0}^{T-y} N(y, c+y) d c}-\frac{N(y, T)}{\int_{y}^{\omega} N(a, T) d a} .
$$

Equation (12) is the difference between the fractional change over age in the number of people who have ever reached age $y$ and the fractional change over age in the number alive above age $y$. The first term, which is always positive, is the ratio between the number of people at $y$ in year $T$ and the number of people who ever reached age $y$, i.e., $\frac{N(y, T)}{\int_{0}^{T-y} N(y, c+y) d c}$, plus the average mortality rate at $y$, weighted by the population 
ever reached age $y$, or $\int_{0}^{T-y} \frac{N(y, c+y)}{\int_{0}^{T-y} N(y, t+y) d t} \mu(y, c+y) d c$. The second term, which is always negative, is the proportion of people age $y$ exactly among all age $y+$ in year $T$. A priori, the sign of Equation (12) is ambiguous. Higher ages imply a greater contribution of mortality on $\pi(y, T)$ due to the positive correlation between age and mortality. But higher ages also imply a greater proportion of people age $y$ among all age $y+$ in the same year.

Figure 5: $\quad$ Fraction of people above alternative threshold ages who ever lived who are alive in year $T$

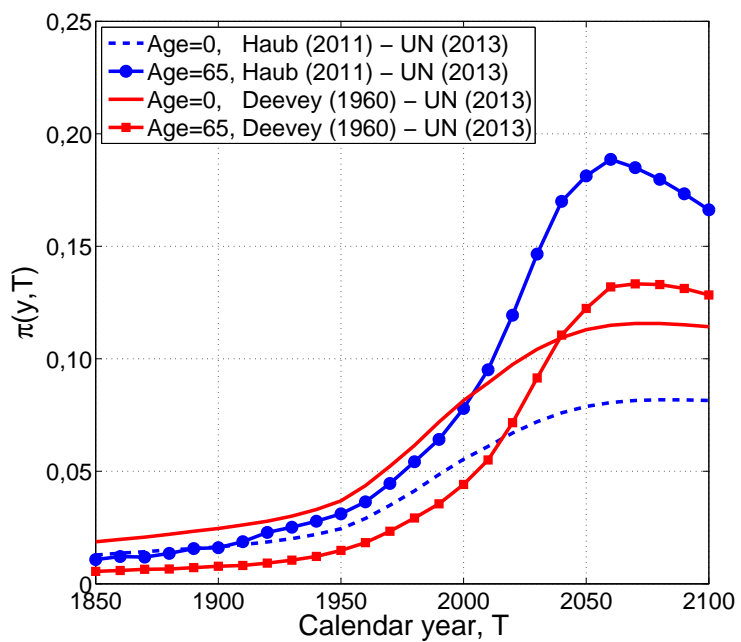

The sign of (12) is, nonetheless, known for some special cases. For example, in a stable population, Proposition 2 shows that $\pi(y, T)$ is monotonically decreasing with respect to the age threshold $y$ (see proof in Appendix B).

Proposition 2. In a stable population with $r>0, \pi(y, T)$ is monotonically decreasing with respect to the age threshold $y$ if the death rate from age $y$ onwards is nondecreasing.

Proposition 2 implies that, in a stable population with $r>0$, the reduction in the number of people alive at age $y$ and older is, in relative terms, smaller than the reduction in the number of people who ever reached age $y$ if, and only if, the death rate from age $y$ onwards is nondecreasing. Therefore, in a growing stable population, $\pi(y, T)$ is increasing early in life, due to the fact that infant death rates are historically higher than the proportion of people at age $y$ ( $y$ belonging to infant ages) among all $y+$; it reaches 
Sánchez-Romero et al.: How many old people have ever lived?

a maximum and it monotonically decreases until very old ages (see Figure 3 in Johnson (1999) for an illustration with a constant population growth rate).

In reality, however, the population growth rate is not constant over time. As in Equation (30), the population growth rate is driven by gains or losses in life expectancy and by increases or decreases in the fertility rate. In this setting, Proposition 2 does not necessarily hold, and it is necessary to perform an empirical analysis. Figure 6 shows, for the two extreme cases modeled with the GIP method, the decomposition of the fractional change over age in the fraction of people above age $y$ ever lived who are alive in 2010.

Figure 6: Decomposition of the fractional change over age in the ratio between the number of people above age $y$ ever lived who are alive in 2010

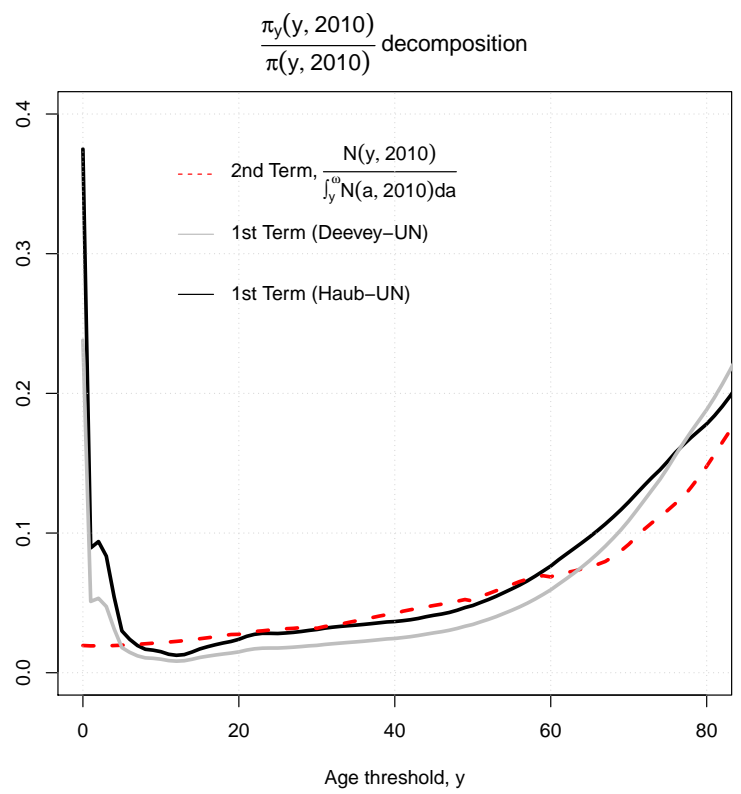

The solid lines (black for Haub-UN and gray for Deevey-UN) represent the fractional change over age in the number of people who ever reached age $y$ (or the first term in Equation (12)), while the dashed red line is the fractional change over age in the number of people alive in 2010 above age $y$ (or the second term in Equation (12)). The second term is the same in both cases since it is based on current population data. In contrast, the black solid line and the gray solid line differ because they are based on historical estimates. Consequently, since historically the age-specific mortality rates are higher in 
Haub (2011) than in Deevey (1960), the black solid line is higher than the gray solid line. Recall that Haub (2011) starts with a life expectancy at birth of age 13, while Deevey (1960) assumes, similar to Keyfitz (1966), a life expectancy of 25 at the onset of Homo sapiens. The crossing point between the gray and black solid lines at old age is due to the higher weight of historical data in Deevey (1960) than in Haub (2011), since the former assumed that more people reached old age. From Figure 6, we know that Equation (12) is positive at young and old ages, i.e., when the solid lines are above the dashed line, and it is negative from age 7 to the end of prime working age (around age 60). Therefore, according to Figure 6 , the fraction $\pi(y, 2010)$ should have a local maximum early in life and a local minimum late in life.

Figure 7: $\quad$ Fraction of people above age $y$ ever lived who were alive in 2010

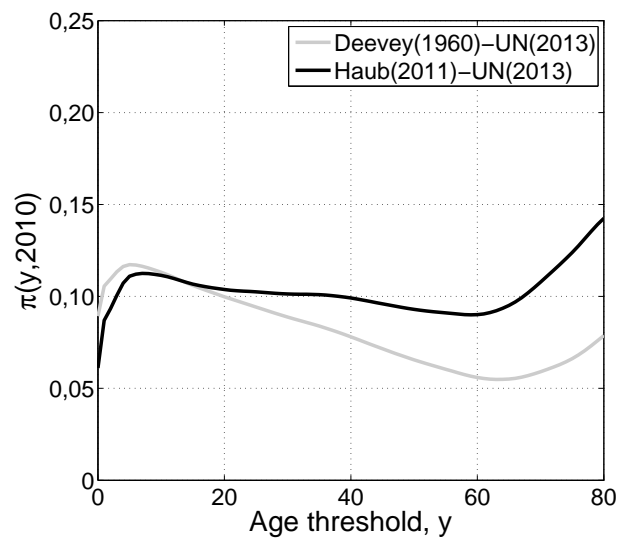

Figure 7 shows the fraction of people above different ages $y$ who ever lived and were alive in 2010 (actual numbers are summarized in Table A-2 in Appendix D). The black solid line depicts $\pi(y, 2010)$ under the assumptions and data of Haub (2011) - UN (2013), while the gray solid line corresponds to that of Deevey (1960) - UN (2013). As Figure 7 suggests, in both cases we find that $\pi(y, 2010)$ increases early in life, reaching a maximum between $11 \%$ and $13 \%$ at age 5 (gray line) and at age 7 (black line). Then, it declines until age 65 (gray line) and age 60 (black line), and finally rises, reaching a value of $8 \%$ (gray line) and almost $15 \%$ (black line) at age 80 . Initially, $\pi(y, 2010)$ rises because the historical average mortality rate at age 0 - i.e., the first term in (12) - until 2010 is close to 23\% (in Deevey - UN) and 35\% (in Haub - UN), while the proportion of recently born among the total population in 2010 is close to $2 \%$. Second, the faster decrease over age in the gray solid line from age 8 to age 60 compared to the black solid line is explained by the lower mortality rate in the former case relative to the proportion 
Sánchez-Romero et al.: How many old people have ever lived?

of people at age $y$ among all age $y+$ in 2010 (cf. Figures 6 and 7). As a consequence, $\pi(65,2010)$ is three percentage points greater in the black solid line (9\%) than in the gray solid line (6\%). Therefore, according to Figure 7 we cannot expect - based on realistic scenarios $-\pi(65,2010)$ values close to $50 \%$ for any age threshold $y<80 .^{11}$

\section{Conclusion and discussion}

The question of how many people have ever lived has been discussed extensively in the demographic literature. In a recent study Cohen (2014) followed this earlier research and studied the change over time in the fraction of people ever born who are currently alive. In this paper, we extend the analysis by Cohen and investigate the fraction of people above a specific age threshold $y$ alive at time $T$ to the population that ever was alive and reached this age threshold, which we denote by $\pi(y, T)$. Such a measure may yield a new view on the pace of population ageing over time. Through our analysis we can show that the guess of Fred Pearce (Economist 2014), that half of all people who have ever reached the age of 65 are alive today, is not true. Indeed, such a number would be never attainable, neither theoretically (in a stable population), nor empirically according to existing data.

Since the stable population model is quite a restrictive approximation over such a long time period, we extended our analysis to a hyperbolic growth model and a nonstable population model where we indirectly estimated the time series of fertility and mortality allowing for differences across various subperiods. Our estimates for the fraction $\pi(65,2010)$ ranges from $5.5 \%$ to about $9.5 \%$, which is clearly well below the estimates cited in Pearce (Economist 2014).

We have applied simple mathematical demography to analytically express $\pi(y, T)$ and use the framework of the Lexis diagram to illustrate this fraction. Assuming a stable population model and a hyperbolic growth model, we were able to derive analytical expressions for $\pi(y, T)$. For the specific case of a stationary population this fraction converges to 0 for $T$ going to infinity. Assuming, however, a stable population with positive growth rate $r>0$ we could analytically derive an expression of the fraction $\pi(y, T)$ which amounts to a weighted integral of the further life expectancy at age $y$ with the weights being an exponential discount with the stable population growth rate.

In the rest of the paper we studied the sensitivity of the fraction $\pi(y, T)$ with respect to the time $T$ and the age $y$.

The fraction $\pi(y, T)$ may be nonmonotonic with respect to $T$, as we have demonstrated in our numerical calculations for values of $\pi(y, T)$ for $T$ between 1850 and 2100 in the case of $y=65$. In this case, $\pi(y, T)$ first increases with $T$, while it decreases

\footnotetext{
${ }_{11}$ Values of $\pi(y, 2010)$ for $y>80$ are not shown because of lack of data above age 80 for the period 19501990. Nevertheless, based on data for living super-centenarians the fraction $\pi$ for supercentenarians in 2000 seems to be close to $12 \%$ (see http://www.grg.org/Adams/E.HTM).
} 
afterwards starting at time periods around $T=2050$. The behavior of $\pi(y, T)$ over time is explained by two terms. The first one is the crude growth rate in year $T$ of the population older than age $y$, which can be either positive or negative. The second one is the fractional change over time in the number of people who ever reached age $y$. During the $20^{t h}$ century and first half of the $21^{\text {st }}$ century the first term will typically be higher than the second one when the growth rate of births increases, because the population reaching age $y$ increases faster than the deaths above that age. The values obtained for various time periods and different age thresholds are again well below $50 \%$ and could be as low as $1 \%$ for early time periods $T=1850$ and up to about $20 \%$ in 2050 .

For illustrations we also provided the range of values for $\pi(65, T)$ for extreme values of $T$ given a stable population under various growth rates of births and for alternative values of the life expectancy at birth. Only in case of a very high growth rate of births could we obtain values of $\pi(65, T)$ similar to the 50\% of Pearce (Economist 2014) or even larger. Nevertheless, using our estimates of population over time, Table 4 shows that the value of $\pi(65,2010)$ is always lower than $50 \%$ even when we start counting the population ever lived to age 65 at more recent years.

Table 4: $\quad$ Estimates of $\pi(65,2010)$ according to the starting year

\begin{tabular}{lccccccc}
\hline Starting year & 4000 B.C & 850 B.C & 0 & 1857 & 1865 & 1900 & 1965 \\
\hline Deevey (1960) & - & $10 \%$ & $11 \%$ & $25 \%$ & - & $28.3 \%$ & $43.5 \%$ \\
Haub (2011) & $10 \%$ & - & $13 \%$ & - & $25 \%$ & $27.5 \%$ & $44.0 \%$ \\
\hline
\end{tabular}

For a given contemporaneous time $T$, we also demonstrated that the fraction is nonmonotonic in age $y$. It first increases with the age threshold at younger ages, then starts to decline before it increases again for older ages. This property can be explained by two opposite forces. The first one is positive and depends on the average historical mortality rate at age $y$. The second is negative and it is the proportion of people at age $y$ among all people age $y+$ in year $T$, which depends on contemporaneous data. The nondecreasing property of $\pi(y, T)$ over the age threshold at young and old ages is explained by the fact that the high mortality rates at these ages in the past dominate over the present mortality rates at these two life periods. Nevertheless, and despite $\pi(y, T)$ increasing at old ages, our results clearly indicate for all age thresholds the value of the fraction $\pi(y, T)$ in year $T=2010$ is far below $50 \%$ and ranges from 0.05 to at most about 0.15 .

Summing up through our analytical and numerical derivations, and by applying realistic time series of historic and future fertility and mortality patterns, we offer realistic estimates of the fraction of people alive today above a specific age among all those who ever lived to this specific age. Our results indicate that this fraction for age 65 has increased over time, supporting the argument that the pace of ageing has increased. 
Sánchez-Romero et al.: How many old people have ever lived?

\section{Acknowledgements}

We thank Joel Cohen, Ronald Lee, Wolfgang Lutz, Marc Luy, Samir K.C., and participants in the 2015 Annual Meeting of the Population Association of America and the Wittgenstein Centre 2016 Conference (Variations of the Themes of Wolfgang Lutz) for their comments and suggestions. 


\section{References}

Coale, A.J. and Zelnik, M. (1963). New estimates of fertility and population in the United States. Princeton: Princeton University Press. doi:10.1515/9781400874934.

Cohen, J.E. (2014). Is the fraction of people ever born who are currently alive rising or falling? Demographic Research 30(56): 1561-1570. doi:10.4054/DemRes.2014.30.56.

Deevey, E.S. (1960). The human population. Scientific American 203(9): 195-204. doi:10.38/scientificamerican0960-194.

Desmond, A. (1962). How many people have ever lived on earth? Population Bulletin 18(1): 1-19.

Economist (2014). Demography, growth and inequality: Age invaders. [electronic resource]. London: The Economist Newspaper. http://www.economist.com/node/21601248/print.

Feichtinger, G. (1979). Demographische Analyse und populationsdynamische Modelle: Grundzüge der Bevölkerungsmathematik. Wien: Springer. doi:10.1007/978-3-70918559-9.

Feichtinger, G. and Vogelsang, H. (1978). Population dynamics with declining fertility. Wien: Institut für Unternehmensforschung der Technischen Hochschule Wien. (Forschungsbericht 15 und 16).

Fucks, W. (1951). Über die Zahl der Menschen, die bisher gelebt haben. Zeitschrift für die gesamte Staatswissenschaft 107: 440-450.

Haub, C. (2011). How many people have ever lived on earth? [electronic resource]. Washington, D.C.: Population Reference Bureau. http://www.prb.org/Publications/Articles/2011 /HowManyPeopleHaveEverLivedonEarth.aspx.

Johnson, P.D. (1999). Observations on the number of humans that have ever lived. Paper presented at the Annual Meeting of the Population Association of America, New York, March 25-27, 1999.

Kapitza, S.P. (1992). Mathematical model of world population growth. Mathematicheskoe Modelirovanie 4(6): 65-79.

Keyfitz, N. (1966). How many people have lived on the earth? Demography 3(2): 581582. doi:10.2307/2060184.

Keyfitz, N. (1977). Applied mathematical demography. New York: Wiley. 
Sánchez-Romero et al.: How many old people have ever lived?

Keyfitz, N. and Caswell, H. (2005). Applied mathematical demography. New York: Springer.

Kremer, M. (1993). Population growth and technological change: One million B.C. to 1990. Quarterly Journal of Economics 108(3): 681-716. doi:10.2307/2118405.

Lee, R.D. (1966). Inverse projection and back projection: A critical appraisal, and comparative results for England, 1539 to 1871. Population Studies 39(2): 233-248. doi:10.1080/0032472031000141466.

Lee, R.D. and Carter, L.R. (1992). Modeling and forecasting U.S. mortality. Journal of the American Statistical Association 87(419): 659-671. doi:10.1002/for.3980110303.

Oeppen, J. (1993). Back projection and inverse projection: Members of a wider class of constrained projection models. Population Studies 47(2): 245-267. doi:10.1080/0032472031000146996.

Petty, S.W. (1682). Essays on mankind and political arithmetic. New York: The Mershon Company. https://archive.org/details/essaysonmankindp00pett.

Preston, S.H., Heuveline, P., and Guillot, M. (2001). Demography: Measuring and modeling population processes. Oxford: Blackwell.

Tattersall, J. (1996). How many people ever lived? In: Calinger, R. (ed.). Vita mathematica: Historical research and integration with teaching. New York: Mathematical Association of America: 331-337.

United Nations Department of Economic and Social Affairs, Population Division (2013). World population prospects: The 2012 revision, key findings and advance tables. New York: United Nations: 11-111 (Working Paper No. ESA/P/WP.227).

von Foerster, H., Mora, P.M., and Amiot, L.W. (1960). Doomsday: Friday, 13 November, A.D. 2026. Science 132(3436): 1291-1295. doi:10.1126/science.132.3436.1291.

von Hoerner, S.J. (1975). Population explosion and interstellar expansion. Journal of the British Interplanetary Society 28: 691-712.

Westing, A.H. (1981). A note on how many humans that have ever lived. BioScience 31(7): 523-524. doi:10.2307/1308496.

Winkler, W. (1959). Wie viele Menschen haben bisher auf der Erde gelebt? Paper presented at International Population Conference 1959, Vienna. 


\section{Appendix A: Proof of Proposition 1}

Let us assume a stable population whose population growth rate is equal to $r$ and the survival probability to age $x$ for any cohort $c$ is equal to $\ell(x)$. Provided that for a stable population the crude growth rate of the population above any age $y$ is constant and equal to the population growth rate, Equation (11) can be written as

$$
\frac{\pi_{T}(y, T)}{\pi(y, T)}=r-\frac{N(y, T)}{\int_{0}^{T-y} N(y, c+y) d c} .
$$

Since by definition $N(y, T)=B(T-y) \ell(y)=B(0) e^{r(T-y)} \ell(y)$, after rearranging terms and solving the integral, Equation (13) becomes

$$
\frac{\pi_{T}(y, T)}{\pi(y, T)}=r-\frac{r}{1-e^{-r(T-y)}}=-\frac{r e^{-r(T-y)}}{1-e^{-r(T-y)}} .
$$

Note that Equation (14) is always negative for any $r \neq 0$, since $\operatorname{sgn}[r]=\operatorname{sgn}\left[\frac{e^{-r(T-y)}}{1-e^{-r(T-y)}}\right]$ For the extreme case that $r=0$, applying l'Hôpital's rule we have

$$
\lim _{r \uparrow 0} \frac{\pi_{T}(y, T)}{\pi(y, T)}=-\lim _{r \uparrow 0} \frac{e^{-r(T-y)}-r(T-y) e^{-r(T-y)}}{(T-y) e^{-r(T-y)}}=-\frac{1}{T-y}<0 .
$$

We thus conclude that in a stable population, $\frac{\pi_{T}(y, T)}{\pi(y, T)}$ is always negative, which proves Proposition 1.

An illustration of the fractional change over time in a Lexis diagram is provided in Figure A-1. 
Sánchez-Romero et al.: How many old people have ever lived?

Figure A-1: Illustration of change over time of the fraction now alive of those who ever survived to old age

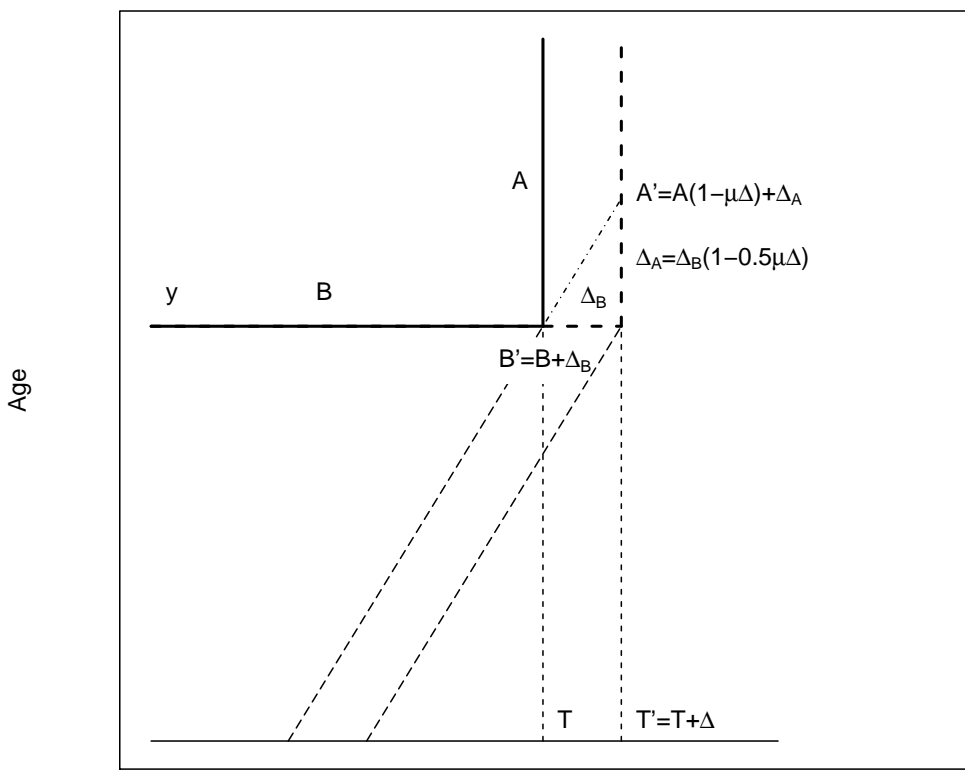

Birth cohorts/Time

\section{Appendix B: Proof of Proposition 2}

Assuming time-constant death rate at age $y\left(\mu_{y}\right)$, let us define

$$
\pi=\frac{A}{B}, \quad \pi^{\prime}=\frac{A^{\prime}}{B^{\prime}},
$$

where

$$
\begin{array}{r}
B^{\prime}=\left(B-\Delta_{B}\right)\left(1-\mu_{y} \Delta\right)=B-\Delta_{B}-B \mu_{y} \Delta+\mu_{y} \Delta_{B} \\
A^{\prime}=A-\Delta_{A}=A-\Delta_{B}+0.5 \mu_{y} \Delta_{B} .
\end{array}
$$


(By contradiction:) if $\pi^{\prime}>\pi$ it should be satisfied that

$$
\frac{A-\Delta_{B}+0.5 \mu_{y} \Delta \Delta_{B}}{B-\Delta_{B}-B \mu_{y} \Delta+\mu_{y} \Delta \Delta_{B}}>\frac{A}{B} .
$$

Rearranging terms and multiplying by -1 gives

$$
\Rightarrow \frac{\Delta_{B}}{A}-0.5 \mu_{y} \Delta \frac{\Delta_{B}}{A}<\frac{\Delta_{B}}{B}+\mu_{y} \Delta-\mu_{y} \Delta \frac{\Delta_{B}}{B} .
$$

Defining $\Delta_{B}=b \Delta$ and simplifying

$$
\Rightarrow \frac{b}{A}-0.5 \mu \Delta \frac{b}{A}<\frac{b}{B}+\mu-\mu \Delta \frac{b}{B} .
$$

Note that $b$ is the total number of births per year who have survived to age $y$, whereas $\Delta$ is an infinitesimal number. Rearranging terms and using the definition of $\pi$ gives

$$
\Rightarrow \frac{b}{A}(1-\pi)<\mu_{y}\left[1+\Delta \frac{b}{A}(0.5-\pi)\right] .
$$

Provided that for any stable population $\lim _{T \rightarrow \infty} \pi=0$, we obtain

$$
\Rightarrow \mu_{y}>\frac{b}{A} \frac{1}{1+0.5 \frac{b}{A} \Delta} .
$$

Under a stationary population $b / A=1 / e_{y}$. Hence,

$$
\Rightarrow \mu_{y}>\frac{1}{e_{y}+0.5 \Delta} .
$$

If the death rate from age $y$ is nondecreasing and $\Delta \rightarrow 0,1 / e_{y} \geq \mu_{y}$, which contradicts the above inequality. Therefore, we have shown that $\pi^{\prime}<\pi$ when the population is stationary.

It is important to realize that $\pi^{\prime}<\pi$ also applies to a stable population with a fixed mortality schedule across cohorts. If the death rate at age $y$ is constant, it can be shown for $\Delta \rightarrow 0$

$$
\begin{aligned}
\Delta_{B}(T) & =\int_{T-\Delta}^{T} N(y, t) d t=\int_{T-\Delta}^{T} B(t-y) \ell(y) d t \\
& =B(T-y) \ell(y) \Delta=N(y, T) \Delta=b(T) \Delta .
\end{aligned}
$$


Sánchez-Romero et al.: How many old people have ever lived?

Figure A-2: Illustration of change over age of the fraction alive now of those who ever survived to old age

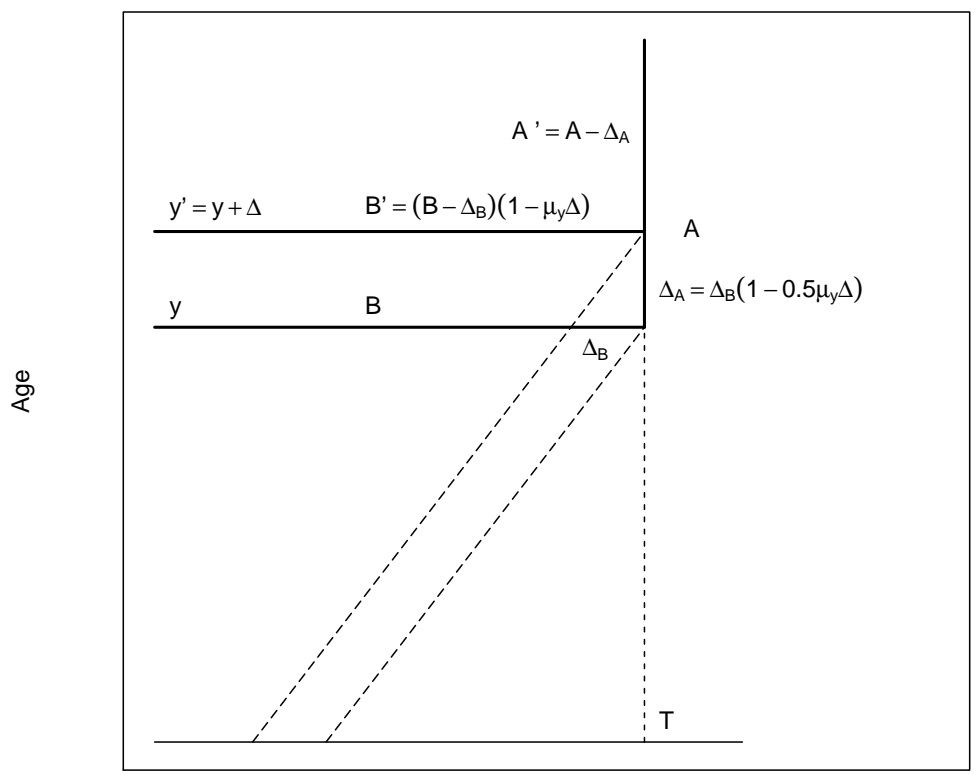

Birth cohorts/Time

Using Equation (30), we have

$$
\begin{aligned}
A(T) & =\int_{y}^{\omega} N(a, T) d a=N(y, T) \int_{y}^{\omega} \frac{N(a, T)}{N(y, T)} d a \\
& =N(y, T) \int_{y}^{\omega} e^{-r(a-y)} \frac{\ell(a)}{\ell(y)} d a=b(T) \int_{y}^{\omega} e^{-r(a-y)} \frac{\ell(a)}{\ell(y)} d a .
\end{aligned}
$$

Therefore, if $r>0$

$$
\frac{b(T)}{A(T)}=\frac{1}{\int_{y}^{\omega} e^{-r(a-y) \frac{\ell(a)}{\ell(y)} d a}}>\frac{1}{e_{y}} \geq \mu_{y},
$$

which also proves by contradiction that $\pi^{\prime}<\pi$. 


\section{Appendix C: Reconstruction of the historical population reported in Table 2 using the GIP method}

To reconstruct the historical population, we use the Generalized Inverse Projection (GIP) model (Lee 1966; Oeppen 1993). The main property of the GIP model is that it gives a population structure that is consistent by age and over time for nonstable populations. This feature is particularly important for reconstructing the change in the population structure after the industrial revolution since the growth rate of the population markedly differs from a constant population growth.

To account for changes in fertility and mortality over time, we consider that the survival probability to age $a$ of an individual born in year $c, \ell(a, c)$, and the fertility rate at age $a$ of an individual born in year $c, f(a, c)$ are, respectively, given by

$$
\begin{aligned}
\ell(a, c) & =e^{-M(a, c)}, \\
f(a, c) & =\left\{\begin{array}{ll}
f \cdot \exp \{\phi(c)\} & \text { if } a=A, \\
0 & \text { otherwise }
\end{array} \text { with } \phi(0)=0,\right.
\end{aligned}
$$

where $M(a, c)=\int_{0}^{a} \mu(x, c+x) d x$ is the cumulative mortality hazard rate at age $a$ for an individual born in year $c$ and $\mu(x, c+x)$ is the mortality hazard rate at age $x$ in year $c+x$. In Equation (29) it is assumed that fertility is concentrated at the mean age at childbearing, where $f$ is the average number of children of the birth cohort $0, \exp \{\phi(c)\}$ indicates the cohort-specific change from the initial cohort in the number of children, and $A$ is the unique age of childbearing.

Like the Lee and Carter (1992) model, we assume that $\log \mu(x, c+x)=\alpha(x)+$ $k(c+x) \beta(x)$, where $\alpha(x)$ and $\beta(x)$ represent the fixed age effects and the rate of change in mortality at age $x$ in response to a change in $k$, and $k(c+x)$ is the level of mortality at time $c+x$. Particular functional forms of Equation (29) have been previously studied in the context of population growth theory. For instance, Coale and Zelnik (1963), Feichtinger and Vogelsang (1978), and Feichtinger (1979) showed that when $\phi(t)=\phi \cdot t$ the birth trajectory is given by $B(t)=B(0) \exp \left\{\frac{\phi}{2} t+\frac{\phi}{2 A} t^{2}\right\}$, where $\phi$ is the rate of change in the level of fertility. Here, however, we assume that total births depend on both fertility and mortality. Thereby, combining (28) and (29) the total number of births born in year $c$ becomes

$$
B(c)=B(0) \exp \left\{\sum_{i=0}^{c / A-1}[\phi(i A)-M(A, i A)]\right\} .
$$

Assuming a unique age of childbearing $(A)$, the renewal equation at time $s+A$ is

$$
B(s+A)=B(s) f(A, s) \ell(A, s) .
$$


Sánchez-Romero et al.: How many old people have ever lived?

From (28) and (29), taking logarithms to both sides of (31) and differentiating with respect to $s$ gives

$$
r(s+A)=r(s)+\phi_{s}(s)-M_{s}(A, s),
$$

where $r(s)$ is the growth rate of births in year $s$. Iterating (32) recursively until time 0 gives

$$
r(s+A) \approx r(0)+\sum_{i=0}^{s / A} \phi_{s}(s-i A)-M_{s}(A, s-i A) .
$$

Integrating (33) with respect to time equals the total contribution of changes in mortality and fertility on the growth rate of births until time $t$ (i.e., $\log \{B(t) / B(0)\}$ )

$$
\int_{0}^{t} r(s) d s \approx r(0) t+\int_{0}^{t} \sum_{i=1}^{s / A} \phi_{s}(s-i A)-M_{s}(A, s-i A) d s .
$$

By changing the order of integration and rearranging terms, we have

$$
\int_{0}^{t} r(s) d s \approx r(0) t+\sum_{i=1}^{t / A} \int_{i A}^{t} \phi_{s}(s-i A)-M_{s}(A, s-i A) d s .
$$

Solving the integral and assuming $r(0) A=\phi(0)-M(A, 0)$ gives

$$
\int_{0}^{t} r(s) d s \approx \sum_{i=0}^{t / A-1} \phi(i A)-M(A, i A) .
$$

which is equivalent to Equation (30).

Equation (30) shows to what extent former changes in fertility and mortality affect the growth rate of births. Substituting (28) and (30) in (3) we get

$$
\pi(y, T)=\frac{\int_{T-\omega}^{T-y} B(c) e^{-M(T-c, c)} d c}{\int_{0}^{T-y} B(c) e^{-M(y, c)} d c} .
$$

Therefore, given $\alpha(x)$ and $\beta(x)$, Equation (34) implies that $\pi(y, T)$ is a function of the history of $\phi(\cdot)$ and $k(\cdot)$.

Our fixed age-specific mortality rates, $\alpha(\cdot)$, as well as the relative rate of change in mortality across age groups, $\beta(\cdot)$, are derived from the model life table by level of life expectancy provided by the UN (2013). Figure A-3 shows the age components of the underlying survival probabilities. These values are calculated taking the first principal component from the mortality data by life expectancy reported by the UN (2013). Moreover, we set the mean age of childbearing $(A)$ at 27, similar to that of Hutterites, given the historical nature of our calculations. 
Figure A-3: Underlying mortality model

(a) Survival probabilities by life expectancy taken from the UN General Model Life Tables

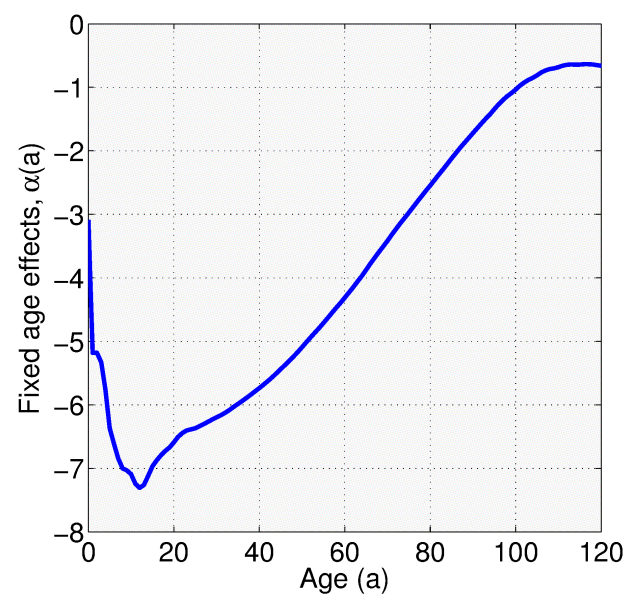

(b)

Age pattern of mortality change, $\beta(a)$

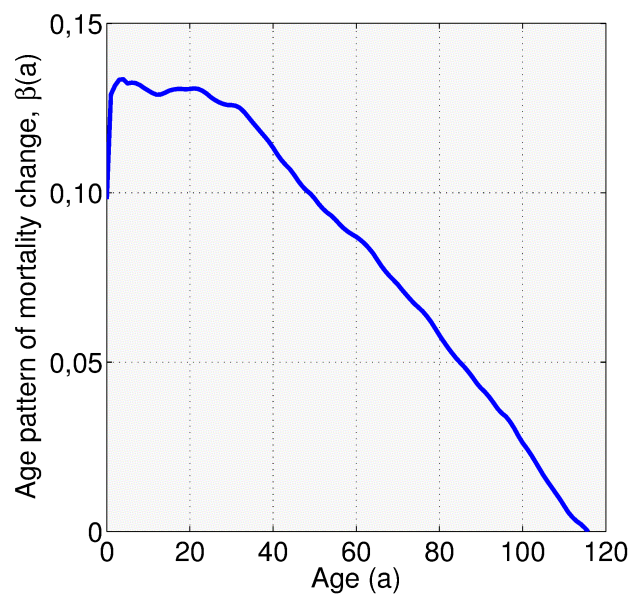


Sánchez-Romero et al.: How many old people have ever lived?

\section{Objective function}

Given an initial number of births $B(0)$ and a time series of demographic values $\left\{N(t), e_{0}(t)\right\}_{t=0}^{T}$ and a set of population distributions $\{N(a, t)\}_{a=0, \ldots, \omega}^{t=0, \ldots, T}$, the historical populations of Haub (2011) and Deevey (1960) are consistently calculated over time by solving the following:

$$
\begin{aligned}
\min _{\mathbf{k}, \boldsymbol{\phi}} F(\mathbf{k}, \boldsymbol{\phi}) & =\sum_{t=0}^{T}\left(\frac{N(t)-\hat{N}(t)}{N(t)}\right)^{2}+\sum_{a=0}^{\omega}\left(\frac{N(a, T)-\hat{N}(a, T)}{N(a, T)}\right)^{2} \\
& +\sum_{t=0}^{T}\left(\frac{B(t)-\hat{B}(t)}{B(t)}\right)^{2}+\sum_{t=0}^{T}\left(\frac{e_{0}(t)-\hat{e}_{0}(t)}{e_{0}(t)}\right)^{2}
\end{aligned}
$$

subject to

$$
\begin{aligned}
\hat{B}(t) & =\hat{B}(t-A) f(A, t-A) \ell(A, t-A), \\
\hat{N}(a, t) & =\hat{B}(t-a) \ell(a, t-a), \\
\hat{N}(t) & =\sum_{a=0}^{\omega} \hat{N}(a, t), \\
\hat{e}_{0}(t) & =\sum_{a=0}^{\omega-1} 0.5[\ell(a, t-a)+\ell(a+1, t-a-1)] \\
\left(I_{2 T} \otimes\left[\begin{array}{c}
1 \\
-1
\end{array}\right]\right)\left[\begin{array}{l}
\mathbf{k} \\
\phi
\end{array}\right] & \leq\left[\begin{array}{llll}
\bar{k} & \bar{\phi} & -\underline{k} & -\phi
\end{array}\right]^{\prime} \otimes 1_{T \times 1},
\end{aligned}
$$

where $\ell(a, t-a)=e^{\left\{-\sum_{s=0}^{a-1} \exp (\alpha(s)+k(t-a+s) \beta(s))\right\}}, f(A, t-A)=f \cdot e^{\phi(t-A)}$, $\mathbf{k}=[k(0), \ldots, k(T)], \phi=[\phi(0), \ldots, \phi(T)]$, and $[\bar{k}, \bar{\phi},-\underline{k},-\phi]$ are the maximum and minimum values of $\{k(t), \phi(t)\}$ for $t=0, \ldots, T$, which are set at $[30,0.5,50,2]$, and $f_{f a b}=0.4886$ is the fraction of female at birth.

Figure A-4 depicts the matching of the GIP method to the UN population distribution for selected years. Although it is almost imperceptible due to the good matching, green solid lines represent the population distribution obtained with the GIP method, and blue solid lines depict UN population data by single years of age.

To derive minimum and maximum values of $\pi(65,2010)$, the values of $\phi(\cdot)$ and $k(\cdot)$ were calculated for the population data of Deevey (1960) and Haub (2011) up to 1900 and the population estimates from 1950 through 2100 reported by the UN (2013). 
Figure A-4: Matching of the GIP method to the UN population distribution, several years from 1950 to 2010

a) Case: Haub (2011)
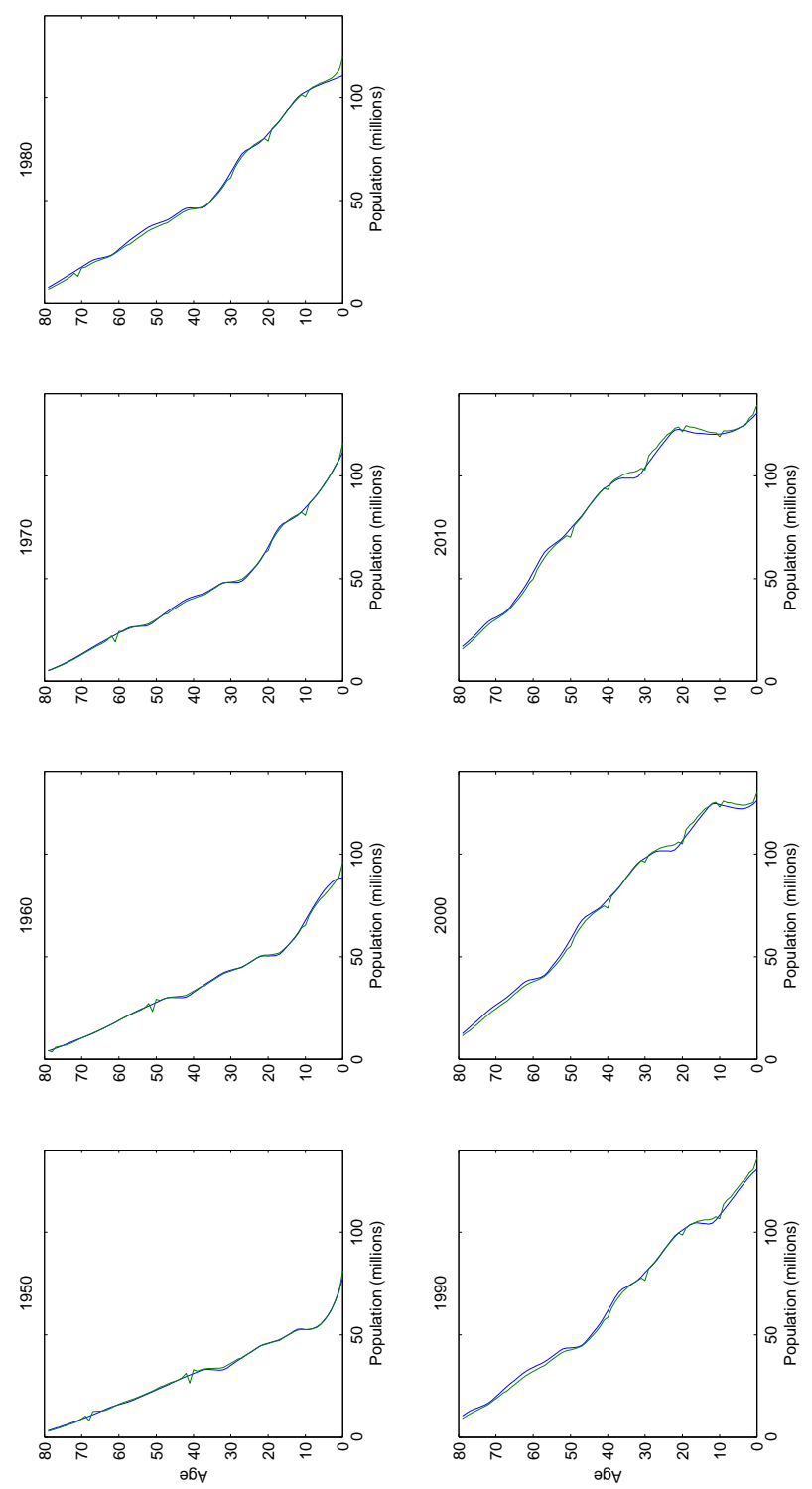
Sánchez-Romero et al.: How many old people have ever lived?

\section{Figure A-4: $\quad$ (Continued)}

b) Case: Deevey (1960)
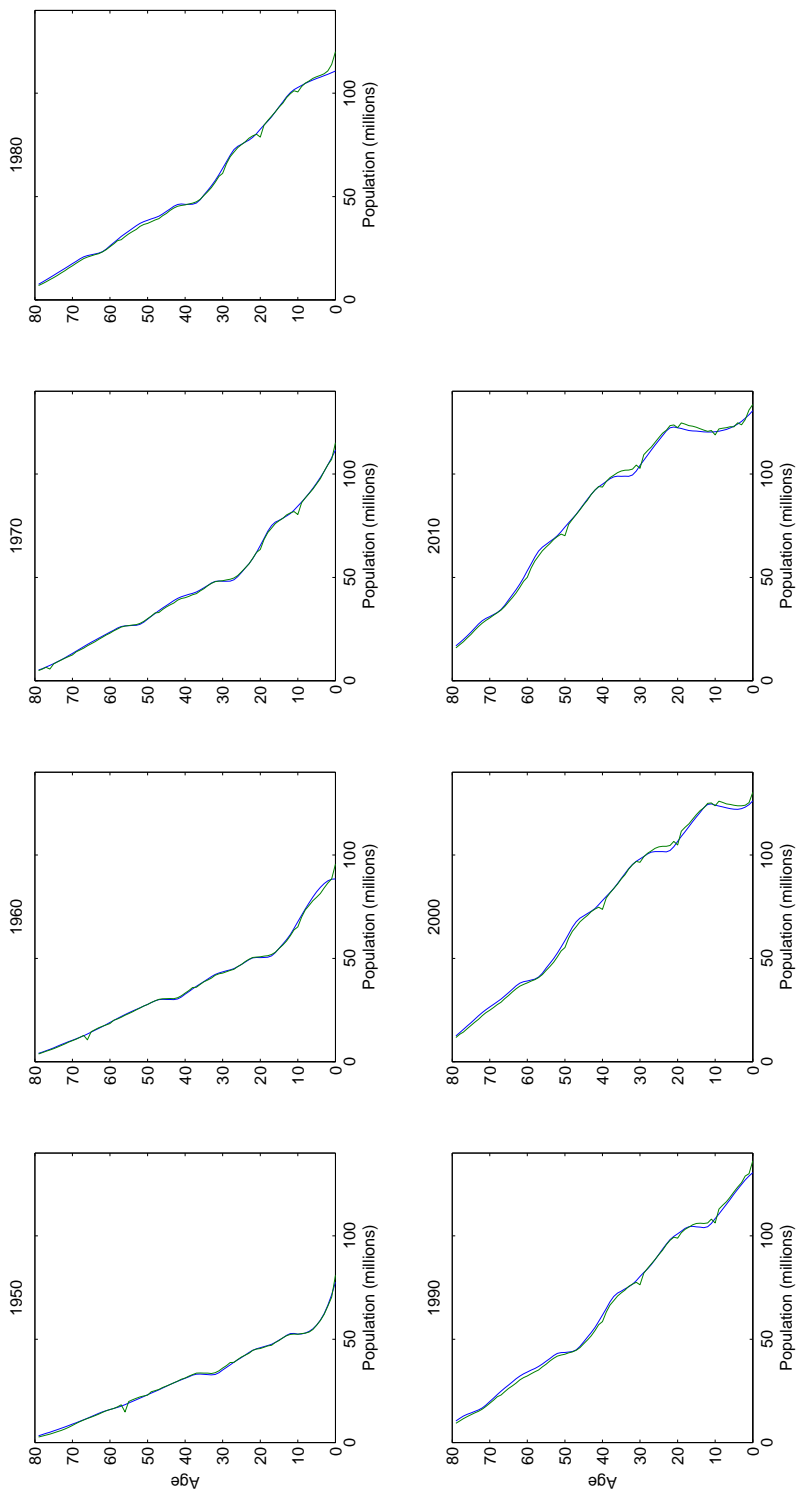


\section{Appendix D: Population ever lived estimates}

Table A-1: Estimates of people ever born by different authors

\begin{tabular}{|c|c|c|c|c|c|c|c|c|c|c|}
\hline $\begin{array}{c}\text { Exact } \\
\text { date }\end{array}$ & Population & Lotka's r & $\begin{array}{l}\text { Crude } \\
\text { birth } \\
\text { rate }\end{array}$ & $\begin{array}{l}\text { Life } \\
\text { expec- } \\
\text { tancy }\end{array}$ & $\begin{array}{l}\text { Births at } \\
\text { exact } \\
\text { date }\end{array}$ & $\begin{array}{l}\text { Births in } \\
\text { period }\end{array}$ & $\begin{array}{l}\text { Cumulated } \\
\text { births }\end{array}$ & $\begin{array}{l}\text { Survival } \\
\text { prob. age } \\
\quad 65\end{array}$ & $\begin{array}{l}\text { Population } \\
\text { ever lived } \\
\text { to age } 65\end{array}$ & $\begin{array}{l}\text { Cumulatec } \\
\text { population } \\
\text { to age } 65\end{array}$ \\
\hline & (millions) & (in \%) & & (years) & (millions) & (millions) & (millions) & & (millions) & (millions \\
\hline \multicolumn{11}{|c|}{ Haub (2011) } \\
\hline-50000 & 0 & 0.035 & 0.080 & 13.0 & 0 & 1,140 & - & & - & \\
\hline-8000 & 5 & 0.051 & 0.080 & 13.0 & 0 & 46,118 & 1,140 & 0.0153 & 17 & 17 \\
\hline 1 & 300 & 0.034 & 0.060 & 17.0 & 18 & 26,614 & 47,259 & 0.0330 & 704 & 721 \\
\hline 1200 & 450 & 0.023 & 0.060 & 17.0 & 27 & 12,813 & 73,872 & 0.0328 & 879 & 1,600 \\
\hline 1650 & 500 & 0.464 & 0.050 & 22.0 & 25 & 3,181 & 86,686 & 0.0671 & 420 & 2,020 \\
\hline 1750 & 795 & 0.464 & 0.040 & 28.0 & 32 & 4,047 & 89,866 & 0.1172 & 213 & 2,234 \\
\hline 1850 & 1,265 & 0.539 & 0.040 & 29.0 & 51 & 2,903 & 93,914 & 0.1226 & 474 & 2,708 \\
\hline 1900 & 1,656 & 0.837 & 0.033 & 38.0 & 55 & 2,986 & 96,817 & 0.2337 & 356 & 3,064 \\
\hline 1945 & 2,516 & & 0.031 & & 78 & & 99,803 & - & 698 & 3,762 \\
\hline
\end{tabular}

Keyfitz (1966)

$\begin{array}{rrrrr}-1000000 & 0 & 0.001 & 0.040 & 25 \\ -5000 & 5 & 0.078 & 0.040 & 25 \\ 0 & 250 & 0.047 & 0.040 & 25 \\ 1650 & 545 & 0.550 & 0.040 & 28 \\ 1945 & 3,000 & - & 0.040\end{array}$

$\begin{array}{rr}25.0 & 0 \\ 25.5 & 0 \\ 25.3 & 10 \\ 28.7 & 22 \\ - & 120\end{array}$

13,508
12,525
24,983
16,121
-

-
13,508
26,034
51,017
67,138

$\begin{array}{rrr}0.0889 & - & - \\ 0.0931 & 1,201 & 1,201 \\ 0.0914 & 1,166 & 2,367 \\ 0.1234 & 2,283 & 4,651 \\ - & 1,989 & 6,640\end{array}$

Westing (1981)

$\begin{array}{rr}-298000 & 0 \\ -40000 & 3 \\ -8000 & 5 \\ 0 & 200 \\ 1650 & 500 \\ 1850 & 1,000 \\ 1945 & 2,300\end{array}$

$\begin{array}{rrrr}0.006 & 0.050 & 20.0 & 0 \\ 0.002 & 0.040 & 25.0 & 0 \\ 0.046 & 0.034 & 30.0 & 0 \\ 0.056 & 0.029 & 35.0 & 6 \\ 0.347 & 0.028 & 40.0 & 14 \\ 0.877 & 0.029 & 45.0 & 29 \\ - & 0.037 & 50.0 & 85\end{array}$

$\begin{array}{rr}2,725 & - \\ 5,014 & 2,725 \\ 14,270 & 7,739 \\ 15,681 & 22,009 \\ 3,992 & 37,690 \\ 4,269 & 41,682 \\ - & 45,951\end{array}$

0.0514
0.0889

0.0889
0.1364

0.1364
0.1929

0.2576

0.3292

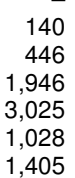

140

586

2,532

5,557

6,585

7,991

Deevey (1960)

\begin{tabular}{rrrrrrrrrrr}
-998040 & 0 & $0.000 \%$ & 0.040 & 25.0 & 0 & 11,782 & - & 0.0889 & - & - \\
-298040 & 1 & $0.000 \%$ & 0.040 & 25.0 & 0 & 21,344 & 11,782 & 0.0889 & 1,048 & 1,048 \\
-23040 & 3 & $0.003 \%$ & 0.040 & 25.0 & 0 & 2,552 & 33,126 & 0.0889 & 1,898 & 2,945 \\
-8040 & 5 & $0.070 \%$ & 0.040 & 25.4 & 0 & 4,658 & 35,678 & 0.0927 & 227 & 3,172 \\
-4040 & 87 & $0.011 \%$ & 0.035 & 28.7 & 3 & 15,132 & 40,336 & 0.1228 & 432 & 3,604 \\
-40 & 133 & $0.083 \%$ & 0.035 & 29.2 & 5 & 17,278 & 55,468 & 0.1282 & 1,858 & 5,462 \\
1650 & 545 & $0.290 \%$ & 0.035 & 30.9 & 19 & 2,212 & 72,746 & 0.1457 & 2,215 & 7,677 \\
1750 & 728 & $0.437 \%$ & 0.035 & 32.2 & 25 & 1,424 & 74,958 & 0.1597 & 322 & 7,999 \\
1800 & 906 & $0.575 \%$ & 0.035 & 33.3 & 32 & 4,286 & 76,383 & 0.1729 & 227 & 8,227 \\
1900 & 1,610 & $0.798 \%$ & 0.035 & 35.5 & 56 & 3,051 & 80,668 & 0.1990 & 741 & 8,968 \\
1945 & - & - & 0.035 & - & - & - & 83,719 & - & 607 & 9,575 \\
\hline \hline
\end{tabular}

Source: Researchers' own calculations based on Deevey (1960), Keyfitz (1966), Westing (1981), and Haub (2011). 
Sánchez-Romero et al.: How many old people have ever lived?

Table A-2: Results of population reconstruction for 2010 and estimates of $\pi$ $(y, 2010)$

\begin{tabular}{|c|c|c|c|c|c|c|}
\hline \multirow{2}{*}{$\begin{array}{l}\text { Age } \\
\\
y\end{array}$} & \multicolumn{2}{|c|}{$\begin{array}{l}\text { Pop. ever lived } \\
\text { up to } 2010 \\
\text { (in millions) }\end{array}$} & \multirow{2}{*}{$\begin{array}{c}\text { Pop. age } y \\
\text { in } 2010 \\
\text { (in millions) } \\
\text { UN, Pop. Div. }\end{array}$} & \multirow{2}{*}{$\begin{array}{l}\text { Pop. age } y+ \\
\text { in } 2010 \\
\text { (in millions) } \\
\text { UN, Pop. Div. }\end{array}$} & \multicolumn{2}{|c|}{$\pi(y, 2010)$} \\
\hline & Haub (2011) & Deevey (1960) & & & Haub (2011) & Deevey (1960) \\
\hline 0 & 112,931 & 77,249 & 131 & 6,896 & 0.0611 & 0.0893 \\
\hline 1 & 77,801 & 64,083 & 129 & 6,765 & 0.0870 & 0.1056 \\
\hline 2 & 71,110 & 60,629 & 127 & 6,637 & 0.0933 & 0.1095 \\
\hline 3 & 64,721 & 57,401 & 125 & 6,510 & 0.1006 & 0.1134 \\
\hline 4 & 59,550 & 54,903 & 124 & 6,384 & 0.1072 & 0.1163 \\
\hline 5 & 56,411 & 53,371 & 123 & 6,260 & 0.1110 & 0.1173 \\
\hline 6 & 54,743 & 52,431 & 122 & 6,137 & 0.1121 & 0.1170 \\
\hline 7 & 53,447 & 51,677 & 122 & 6,015 & 0.1125 & 0.1164 \\
\hline 8 & 52,414 & 51,049 & 121 & 5,893 & 0.1124 & 0.1154 \\
\hline 9 & 51,536 & 50,487 & 121 & 5,772 & 0.1120 & 0.1143 \\
\hline 10 & 50,707 & 49,953 & 120 & 5,651 & 0.1114 & 0.1131 \\
\hline 11 & 49,945 & 49,465 & 120 & 5,531 & 0.1107 & 0.1118 \\
\hline 12 & 49,286 & 49,025 & 120 & 5,410 & 0.1098 & 0.1104 \\
\hline 13 & 48,672 & 48,597 & 120 & 5,290 & 0.1087 & 0.1089 \\
\hline 14 & 48,039 & 48,143 & 121 & 5,170 & 0.1076 & 0.1074 \\
\hline 15 & 47,327 & 47,635 & 121 & 5,049 & 0.1067 & 0.1060 \\
\hline 16 & 46,522 & 47,075 & 121 & 4,928 & 0.1059 & 0.1047 \\
\hline 17 & 45,658 & 46,478 & 121 & 4,808 & 0.1053 & 0.1034 \\
\hline 18 & 44,747 & 45,849 & 122 & 4,687 & 0.1047 & 0.1022 \\
\hline 19 & 43,798 & 45,195 & 122 & 4,565 & 0.1042 & 0.1010 \\
\hline 20 & 42,824 & 44,514 & 122 & 4,443 & 0.1038 & 0.0998 \\
\hline 21 & 41,803 & 43,796 & 123 & 4,321 & 0.1034 & 0.0987 \\
\hline 22 & 40,721 & 43,038 & 122 & 4,198 & 0.1031 & 0.0975 \\
\hline 23 & 39,611 & 42,259 & 121 & 4,076 & 0.1029 & 0.0964 \\
\hline 24 & 38,503 & 41,481 & 119 & 3,955 & 0.1027 & 0.0953 \\
\hline 25 & 37,428 & 40,715 & 116 & 3,836 & 0.1025 & 0.0942 \\
\hline 26 & 36,387 & 39,960 & 114 & 3,720 & 0.1022 & 0.0931 \\
\hline 27 & 35,363 & 39,206 & 112 & 3,606 & 0.1020 & 0.0920 \\
\hline 28 & 34,352 & 38,452 & 109 & 3,494 & 0.1017 & 0.0909 \\
\hline 29 & 33,350 & 37,694 & 107 & 3,385 & 0.1015 & 0.0898 \\
\hline 30 & 32,351 & 36,931 & 104 & 3,279 & 0.1013 & 0.0888 \\
\hline 31 & 31,359 & 36,167 & 101 & 3,174 & 0.1012 & 0.0878 \\
\hline 32 & 30,372 & 35,399 & 100 & 3,073 & 0.1012 & 0.0868 \\
\hline 33 & 29,396 & 34,630 & 99 & 2,973 & 0.1012 & 0.0859 \\
\hline 34 & 28,436 & 33,862 & 99 & 2,875 & 0.1011 & 0.0849 \\
\hline 35 & 27,493 & 33,096 & 99 & 2,776 & 0.1010 & 0.0839 \\
\hline 36 & 26,571 & 32,332 & 99 & 2,677 & 0.1007 & 0.0828 \\
\hline 37 & 25,665 & 31,570 & 99 & 2,578 & 0.1004 & 0.0817 \\
\hline 38 & 24,776 & 30,808 & 98 & 2,479 & 0.1001 & 0.0805 \\
\hline 39 & 23,903 & 30,048 & 96 & 2,381 & 0.0996 & 0.0793 \\
\hline 40 & 23,047 & 29,290 & 95 & 2,285 & 0.0992 & 0.0780 \\
\hline 41 & 22,213 & 28,539 & 94 & 2,190 & 0.0986 & 0.0767 \\
\hline 42 & 21,398 & 27,791 & 92 & 2,096 & 0.0980 & 0.0754 \\
\hline 43 & 20,599 & 27,042 & 90 & 2,004 & 0.0973 & 0.0741 \\
\hline 44 & 19,811 & 26,289 & 88 & 1,914 & 0.0966 & 0.0728 \\
\hline 45 & 19,030 & 25,532 & 85 & 1,826 & 0.0960 & 0.0715 \\
\hline 46 & 18,261 & 24,775 & 83 & 1,741 & 0.0953 & 0.0703 \\
\hline 47 & 17,508 & 24,018 & 81 & 1,658 & 0.0947 & 0.0690 \\
\hline 48 & 16,765 & 23,257 & 78 & 1,577 & 0.0941 & 0.0678 \\
\hline 49 & 16,029 & 22,488 & 76 & 1,499 & 0.0935 & 0.0666 \\
\hline 50 & 15,296 & 21,709 & 74 & 1,422 & 0.0930 & 0.0655 \\
\hline 51 & 14,572 & 20,927 & 72 & 1,348 & 0.0925 & 0.0644 \\
\hline
\end{tabular}


Table A-2: $\quad$ (Continued)

\begin{tabular}{|c|c|c|c|c|c|c|}
\hline \multirow{2}{*}{$\begin{array}{l}\text { Age } \\
\\
y\end{array}$} & \multicolumn{2}{|c|}{$\begin{array}{l}\text { Pop. ever lived } \\
\text { up to } 2010 \\
\text { (in millions) }\end{array}$} & \multirow{2}{*}{$\begin{array}{c}\text { Pop. age y } \\
\text { in } 2010 \\
\text { (in millions) } \\
\text { UN, Pop. Div. }\end{array}$} & \multirow{2}{*}{$\begin{array}{l}\text { Pop. age } y+ \\
\text { in } 2010 \\
\text { (in millions) } \\
\text { UN, Pop. Div. }\end{array}$} & \multicolumn{2}{|c|}{$\pi(y, 2010)$} \\
\hline & Haub (2011) & Deevey (1960) & & & Haub (2011) & Deevey (1960) \\
\hline 52 & 13,855 & 20,136 & 70 & 1,276 & 0.0921 & 0.0634 \\
\hline 53 & 13,146 & 19,338 & 69 & 1,206 & 0.0917 & 0.0623 \\
\hline 54 & 12,444 & 18,532 & 67 & 1,137 & 0.0914 & 0.0613 \\
\hline 55 & 11,748 & 17,719 & 66 & 1,070 & 0.0910 & 0.0604 \\
\hline 56 & 11,062 & 16,901 & 65 & 1,004 & 0.0907 & 0.0594 \\
\hline 57 & 10,387 & 16,078 & 63 & 939 & 0.0904 & 0.0584 \\
\hline 58 & 9,723 & 15,252 & 60 & 876 & 0.0901 & 0.0574 \\
\hline 59 & 9,071 & 14,423 & 56 & 816 & 0.0900 & 0.0566 \\
\hline 60 & 8,432 & 13,592 & 53 & 760 & 0.0901 & 0.0559 \\
\hline 61 & 7,810 & 12,763 & 50 & 707 & 0.0905 & 0.0554 \\
\hline 62 & 7,202 & 11,936 & 47 & 657 & 0.0912 & 0.0550 \\
\hline 63 & 6,615 & 11,118 & 44 & 610 & 0.0922 & 0.0549 \\
\hline 64 & 6,052 & 10,313 & 42 & 566 & 0.0935 & 0.0549 \\
\hline 65 & 5,514 & 9,524 & 39 & 524 & 0.0951 & 0.0551 \\
\hline 66 & 5,003 & 8,754 & 37 & 485 & 0.0970 & 0.0554 \\
\hline 67 & 4,520 & 8,006 & 34 & 449 & 0.0993 & 0.0561 \\
\hline 68 & 4,065 & 7,282 & 33 & 414 & 0.1019 & 0.0569 \\
\hline 69 & 3,638 & 6,585 & 32 & 381 & 0.1048 & 0.0579 \\
\hline 70 & 3,239 & 5,918 & 31 & 349 & 0.1078 & 0.0590 \\
\hline 71 & 2,868 & 5,282 & 30 & 318 & 0.1109 & 0.0602 \\
\hline 72 & 2,524 & 4,680 & 29 & 288 & 0.1141 & 0.0615 \\
\hline 73 & 2,208 & 4,117 & 27 & 259 & 0.1172 & 0.0629 \\
\hline 74 & 1,921 & 3,593 & 25 & 231 & 0.1204 & 0.0644 \\
\hline 75 & 1,662 & 3,113 & 24 & 206 & 0.1239 & 0.0661 \\
\hline 76 & 1,430 & 2,674 & 22 & 182 & 0.1275 & 0.0682 \\
\hline 77 & 1,222 & 2,276 & 20 & 161 & 0.1314 & 0.0705 \\
\hline 78 & 1,039 & 1,922 & 18 & 141 & 0.1354 & 0.0732 \\
\hline 79 & 878 & 1,610 & 17 & 122 & 0.1391 & 0.0759 \\
\hline 80 & 739 & 1,338 & 15 & 105 & 0.1425 & 0.0787 \\
\hline 81 & 618 & 1,103 & 14 & 90 & 0.1452 & 0.0814 \\
\hline 82 & 514 & 901 & 13 & 76 & 0.1473 & 0.0841 \\
\hline 83 & 425 & 730 & 11 & 63 & 0.1487 & 0.0866 \\
\hline 84 & 348 & 585 & 10 & 52 & 0.1495 & 0.0890 \\
\hline 85 & 283 & 463 & 8 & 42 & 0.1496 & 0.0914 \\
\hline 86 & 228 & 363 & 7 & 34 & 0.1492 & 0.0936 \\
\hline 87 & 181 & 280 & 6 & 27 & 0.1484 & 0.0959 \\
\hline 88 & 143 & 214 & 5 & 21 & 0.1470 & 0.0979 \\
\hline 89 & 111 & 162 & 4 & 16 & 0.1449 & 0.0996 \\
\hline 90 & 86 & 121 & 3 & 12 & 0.1419 & 0.1005 \\
\hline 91 & 65 & 89 & 2 & 9 & 0.1387 & 0.1012 \\
\hline 92 & 49 & 65 & 2 & 7 & 0.1367 & 0.1029 \\
\hline 93 & 36 & 46 & 1 & 5 & 0.1367 & 0.1059 \\
\hline 94 & 26 & 33 & 1 & 4 & 0.1379 & 0.1098 \\
\hline 95 & 19 & 23 & 1 & 3 & 0.1390 & 0.1135 \\
\hline 96 & 13 & 16 & 1 & 2 & 0.1403 & 0.1174 \\
\hline 97 & 9 & 11 & 0 & 1 & 0.1391 & 0.1191 \\
\hline 98 & 6 & 7 & 0 & 1 & 0.1329 & 0.1160 \\
\hline 99 & 4 & 4 & 0 & 0 & 0.1231 & 0.1093 \\
\hline $100+$ & 7 & 7 & 0 & 0 & & \\
\hline
\end{tabular}


Sánchez-Romero et al.: How many old people have ever lived? 